Article

\title{
Dietary Intake in the Lifelines Cohort Study: Baseline Results from the Flower Food Frequency Questionnaire among 59,982 Participants
}

\author{
A. Mireille Baart*(D), Elske M. Brouwer-Brolsma (D), Corine W. M. Perenboom, Jeanne H. M. de Vries (D) \\ and Edith J. M. Feskens (D)
}

Citation: Baart, A.M.;

Brouwer-Brolsma, E.M.;

Perenboom, C.W.M.; de Vries, J.H.M.; Feskens, E.J.M. Dietary Intake in the Lifelines Cohort Study: Baseline Results from the Flower Food Frequency Questionnaire among 59,982 Participants. Nutrients 2022, 14, 48. https://doi.org/10.3390/ nu14010048

Academic Editors: Zumin Shi and Jaakko Tuomilehto

Received: 1 November 2021

Accepted: 21 December 2021

Published: 23 December 2021

Publisher's Note: MDPI stays neutral with regard to jurisdictional claims in published maps and institutional affiliations.

Copyright: (c) 2021 by the authors Licensee MDPI, Basel, Switzerland. This article is an open access article distributed under the terms and conditions of the Creative Commons Attribution (CC BY) license (https:// creativecommons.org/licenses/by/ $4.0 /)$.
Division of Human Nutrition and Health, Wageningen University \& Research, 6700 AA Wageningen, The Netherlands; elske.brouwer-brolsma@wur.nl (E.M.B.-B.); corine.perenboom@wur.nl (C.W.M.P.); jeanne.devries@wur.nl (J.H.M.d.V.); edith.feskens@wur.nl (E.J.M.F.)

* Correspondence: mireille.baart@wur.nl; Tel.: +31-(0)317-48-91-43

\begin{abstract}
The role of nutrition in health and disease is well established. However, more research on this topic is needed to fill gaps in our current knowledge. The Lifelines cohort study, a large Dutch prospective cohort study, was established as a resource for international researchers, aiming to obtain insight into the aetiology of healthy ageing. The study started with 167,729 participants, covering three generations, aiming to follow them for thirty years. This article describes the habitual dietary intake, assessed using the Flower Food Frequency Questionnaire (FFQ), among Lifelines cohort study participants at baseline, stratified by sex and different categories of age, socioeconomic status (SES) and body mass index (BMI). A total of 59,982 adults (23,703 men and 36,279 women), who completed the Flower FFQ and reported plausible habitual dietary intake, were included in the analyses. Median daily energy intake was higher in men $(2368 \mathrm{kcal})$ than in women $(1848 \mathrm{kcal})$, as well as macronutrient intake. Energy and macronutrient intake decreased with increasing age and BMI categories; no differences were observed between SES categories. Intake of most micronutrients was higher in men than in women. Differences were observed between age categories, but not between SES and BMI categories. Food groups were consumed in different amounts by men and women; differences between age, SES and BMI categories were observed as well. The Lifelines cohort study provides extensive dietary intake data, which are generalisable to the general Dutch population. As such, highly valuable dietary intake data are available to study associations between dietary intake and the development of chronic diseases and healthy aging.
\end{abstract}

Keywords: dietary intake; micronutrients; macronutrients; food groups; nutrition survey

\section{Introduction}

Nutrition plays an important role in health status, and an unhealthy diet is one of the key determinants of non-communicable morbidity and mortality [1]. For example, dietary patterns consisting of energy-dense, high-fat diets, with low fruit and vegetable intakes, are associated with an increased risk of developing diet-related non-communicable diseases such as cardiovascular diseases, type 2 diabetes and some cancers [2]. Although the existence of a relationship between nutrition and health status is evident, more research on this topic is warranted to fill the gaps in knowledge. For instance, only few well-established clear links between nutrition and cancer exist. Future research might show further important risk factors or protective factors, for example specific food components or broader dietary patterns such as plant-based diets [3]. Regarding type 2 diabetes, several associations between dietary factors and this disease have been reported; however, only few of these associations were graded as high quality of evidence. More well-conducted research, with more detailed assessment of diet, is needed to achieve high quality of evidence for these associations and to be able to give strong dietary recommendations [4]. Moreover, 
in a review article on diet and cardiovascular disease, it was concluded that future research is indispensable for furthering our understanding of the role of diet in this disease and for translating nutritional science into practice [5]. The above applies to many other diseases as well. Furthermore, many interactions with other factors, such as genetic background [6] or gut microbiome [7], have not yet been elucidated. Large epidemiological studies offer the opportunity to further investigate associations between nutrition and health status and interactions with other factors, and to disentangle underlying pathways [8].

The Lifelines cohort study, a multi-disciplinary prospective population-based cohort study in the north of The Netherlands, was established in 2006 as a resource for international researchers, aiming to obtain insight into the aetiology of healthy ageing [9]. It employs a broad range of investigative procedures in assessing the behavioural, socio-demographic, biomedical, physical and psychological factors which contribute to the health and disease of the general population, with a special focus on multi-morbidity and complex genetics. The study started with 167,729 participants, covering three generations, aiming to follow them for at least thirty years. Questionnaires on demographics, health and lifestyle, including dietary intake, are administered every eighteen months, and physical measurements as well as biological sampling are scheduled every five years. With this large body of data, the Lifelines database offers a unique opportunity to study complex interactions between environmental, phenotypic and genomic factors in the development of chronic diseases and healthy ageing, including diet-disease associations.

Within the Lifelines cohort study, data on habitual dietary intake is collected using the Flower Food Frequency Questionnaire (FFQ) [10]. This FFQ was especially developed for the Lifelines cohort study as an alternative to the regular comprehensive FFQ, which is a long and time-consuming questionnaire. The Flower FFQ consists of one main questionnaire and three short complementary questionnaires that are administered at different time points during a five-year period, reducing the time for filling out the questionnaire per occasion and with that participant burden. This is important as participants of the Lifelines cohort study must fill out many other questionnaires and undergo several physical measurements. Based on the literature, stable food consumption patterns over time can be assumed [11]. As such, a valid long-term estimate of the habitual dietary intake of participants is obtained, including data on the intake of energy, several macro- and micronutrients, and food items. Both the breadth of available variables and the large sample size of the Lifelines cohort provide the opportunity to perform well-powered stratified analyses to thoroughly investigate associations between dietary intake and the development of chronic diseases and healthy aging. The purpose of this article is to describe dietary intake among Lifelines cohort study participants at baseline. Data are presented separately for men and women and stratified by different categories of age, socioeconomic status (SES) and body mass index (BMI).

\section{Methods}

\subsection{Study Population}

By the end of 2006, recruitment of participants for the Lifelines cohort study started among inhabitants of the northern three provinces of The Netherlands (Friesland, Groningen and Drenthe). All general practitioner's practices in this area that used computerised patient records (over $80 \%$ of practices) were requested to help with the recruitment. Within these practices, all patients in the age range of 25-50 years were invited by their general practitioner. Exclusion criteria included having a severe mental or physical illness, limited life expectancy ( $<5$ years), and insufficient knowledge of the Dutch language to complete a Dutch questionnaire. Eligible participants received a first questionnaire and were invited to a Lifelines research facility for a comprehensive health assessment. During this visit, participants were also asked to indicate whether family members would be willing to participate in the study, and in case of a positive response, family members were invited as well. Children were only allowed to participate if one of their parents was included in the study. In addition to this recruitment strategy, inhabitants of the three northern provinces 
could also register themselves via the Lifelines website. In December 2013, the recruitment period was closed after reaching the target number of 165,000 participants. At that time, the total number of participants included was 167,729. A more detailed description of the total study population of the Lifelines cohort study can be found elsewhere [9].

The Lifelines cohort study is conducted according to the principles of the Declaration of Helsinki and in accordance with the research code of the University Medical Center Groningen (UMCG). The Lifelines cohort study is approved by the medical ethical committee of the UMCG, the Netherlands. All participants gave written informed consent.

\subsection{Assessment of Dietary Intake}

Dietary intake at baseline was assessed using the validated Flower FFQ (Figure 1) [10]. The name Flower FFQ is derived from its design. The FFQ consists of one main questionnaire, which symbolises the heart of the flower, and three complementary questionnaires, which symbolise the flower petals. The heart FFQ contains 110 food items used to estimate intakes of major food groups, energy, carbohydrates, fat, protein and alcohol, but not in much detail. The three petal FFQs ask for detailed information on the types of food consumed within the food groups of the heart FFQ and also for supplement intake, to be able to estimate the intake of specific (micro)nutrients and food components. Thus, the difference between the heart FFQ and the petal FFQs is the degree of detail requested. For example, the heart FFQ provides basic information about the total amount of bread consumed, without information about the type of bread. More detailed information about bread type is provided by the third FFQ petal, in which the question "Did you eat bread?" is followed by questions on the type of bread (e.g., white, whole wheat). The first petal FFQ contains 59 food items used to estimate intakes of different types of fatty acids and caffeine; the second petal FFQ contains 61 food items used to estimate intakes of B-vitamins, calcium and soy; the third petal FFQ contains 64 food items used to estimate intakes of vitamin A, vitamin C, vitamin E and dietary fibre. Combined, the heart FFQ and the three petal FFQs cover 212 food items.

All adult participants of the Lifelines cohort study were invited to complete the Flower FFQ. The reference period of the heart and petal FFQs is one month, but it is assumed that food consumption patterns are stable over a longer period of time [11]. Therefore, the heart FFQ and the petal FFQs can be filled out at different moments during a study period. At the first assessment (between 2007 and 2013), participants received an invitation to fill out the heart FFQ. During three subsequent assessments (2011-2014, 2012-2015 and 2014-2017), they received an invitation to fill out one of the petal FFQs. The three petal FFQs were randomly distributed to adult participants during these subsequent assessments so that each participant received the petals in one out of six possible orders. Within the four different assessments, participants filled out the FFQs at time points that were fairly evenly distributed over the years and seasons. These four assessments are referred to as the baseline for dietary intake. At future assessments in the coming years, participants will be invited to complete the heart FFQ and the petal FFQs again, which will be referred to as follow-ups for dietary intake. Not all participants completed the four questionnaires. In this article, dietary intake from participants who completed the total Flower FFQ, i.e., the heart FFQ and all three petal FFQs, is described.

With combined data obtained from the heart FFQ and the three petal FFQs, the frequency of consumption of food items was assessed. Questions pertaining to frequency were completed by selecting answers ranging from "never" to "6-7 days per week". Portion sizes were estimated using natural portions and commonly used household measures. In case a food item was reported in either only the heart FFQ or only in the petal FFQ, the food item was considered to be not consumed. 


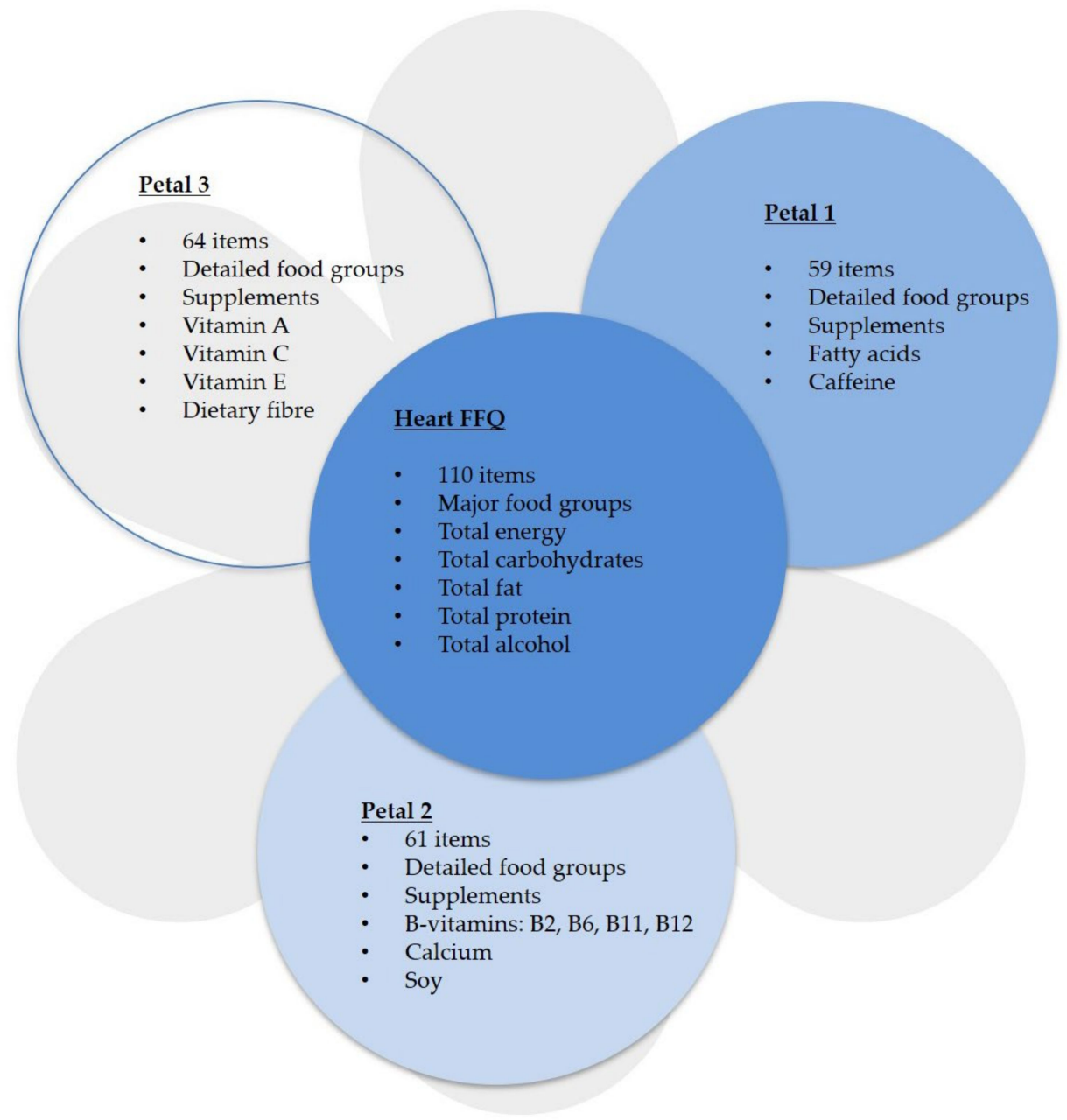

Figure 1. Flower FFQ. FFQ: Food Frequency Questionnaire.

From data on food consumption obtained with the Flower FFQ, average daily intake of foods was calculated. The 212 food items were categorised into 30 food groups (Supplementary Table S1). Data on food consumption were also converted into daily energy and nutrient intake using data from the Dutch food composition database of 2011 [12].

To correct for potential under- or overreporting, we excluded participants with implausible habitual dietary intake, i.e., with energy intake $<800$ and $>4200 \mathrm{kcal}$ for men and $<500$ and $>3500 \mathrm{kcal}$ for women $[13,14]$.

\subsection{Assessment of Other Characteristics}

Data on sex, age, ethnicity, SES, smoking and physical activity were obtained from questionnaires. Age was categorised into age groups used by the Health Council of the Netherlands for the recommendation of nutrients, as follows: 18-50 years, 51-70 years and $>70$ years [15]. SES was categorised based on education attainment, because education is more differentiating than income in the Dutch population [16], as follows: no education, primary education, lower vocational education, lower general secondary education (low); intermediate vocational education, higher general secondary education (moderate); higher vocational education and university education (high). Smoking was categorised as current, former and never smoker and included use of cigarettes, cigarillos, cigars and pipe tobacco. Physical activity was assessed with the short questionnaire to assess health-enhancing physical activity (SQUASH). The SQUASH has been shown to be substantially correlated with physical activity measured by accelerometry (correlation coefficient $=0.45$ ) [17]. Using the SQUASH, the average number of minutes per week of various domains of physical 
activity (e.g., commuting, work, household chores, leisure time including, e.g., gardening and sports) were assessed. Based on Ainsworth's compendium of physical activities [18], metabolic equivalent of task (MET) values were assigned to the specific physical activities. Subsequently, the total number of minutes per week of moderate to vigorous physical activity (MVPA) was calculated, using MET values of $\geq 4.0$ to $<6.5$ for moderate physical activity and MET values $\geq 6.5$ for vigorous physical activity.

Anthropometric measurements were conducted by well-trained staff at Lifelines research facilities. Height and body weight were measured without shoes and heavy clothing. Height was measured using the SECA 222 stadiometer (Seca GmbH, Hamburg, Germany); body weight was measured using the SECA 761 scale (Seca GmbH, Hamburg, Germany). BMI was calculated as $\mathrm{kg} / \mathrm{m}^{2}$ and then categorised into normal weight $\left(\mathrm{BMI}<25 \mathrm{~kg} / \mathrm{m}^{2}\right)$, overweight $\left(25 \leq \mathrm{BMI}<30 \mathrm{~kg} / \mathrm{m}^{2}\right)$ and obesity $\left(\mathrm{BMI} \geq 30 \mathrm{~kg} / \mathrm{m}^{2}\right)$ [19].

\subsection{Statistical Analyses}

Data were first checked for normality using a Kolmogorov-Smirnov test and visual inspection of Q-Q normality plots. All continuous variables showed a skewed distribution and are therefore presented as medians with 25 th-75th percentiles. Categorical variables are presented as numbers with percentages.

To explore potential selection bias, characteristics of participants who completed the total Flower FFQ and who did not complete the total Flower FFQ were compared first, using a Mann-Whitney $U$ test for continuous variables and a Chi-square test for categorical variables, for men and women separately. Thereafter, analyses were performed among participants who completed the total Flower FFQ.

Daily energy and nutrient intake, as well as intake of food groups, were compared between men and women using a Mann-Whitney $U$ test. Dietary intake was also compared between different categories of age, SES and BMI, using a Kruskal-Wallis test, for men and women separately. To identify which categories were different from each other, post hoc pairwise comparisons were performed, using a Bonferroni correction to adjust for multiple testing.

The results of all statistical tests were considered significant when the level of significance was lower than 5\%, i.e., $p<0.05$. Statistical analyses were performed with SPSS software (Version 25, IBM, Armonk, NY, USA).

\section{Results}

Figure 2 presents the participant flow. A total of 144,093 adults completed the heart FFQ. For 15,220 of these participants (11\%), misreporting was highly likely because they reported unlikely low or high energy intake; 128,873 participants (89\%) reported plausible habitual dietary intake. A total of 68,698 adults completed the total Flower FFQ. For 8716 of these participants (13\%), misreporting was highly likely; 59,982 participants (87\%) reported plausible habitual dietary intake. In this article, we primarily focus on the dietary intake data of these 59,982 participants (23,703 men and 36,279 women). Because of the large study population, almost all statistical tests were significant, even if differences were very small. Therefore, only striking and relevant differences are described.

Participants received an invitation to fill out the heart FFQ at the first assessment (between 2007 and 2013). During three subsequent assessments (2011-2014, 2012-2015 and 2014-2017), they received an invitation to fill out one of the petal FFQs. The three petal FFQs were randomly distributed to the participants during the follow-up so that each participant received the petals in one out of six possible orders. 
Year

$\begin{array}{llllllllllll}2006 & 2007 & 2008 & 2009 & 2010 & 2011 & 2012 & 2013 & 2014 & 2015 & 2016 & 2017\end{array}$

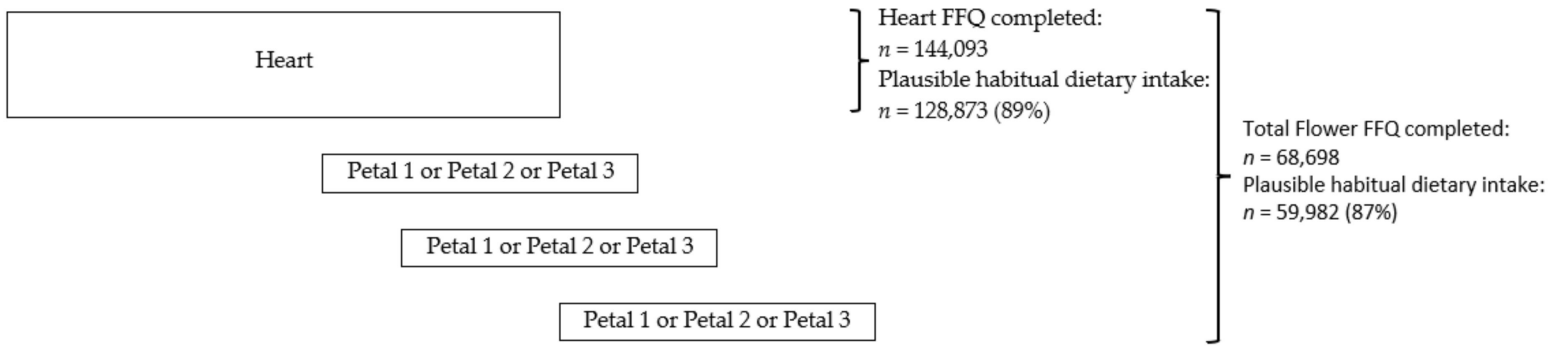

Figure 2. Participant flow and timeline of completing the Flower FFQ.

\subsection{Participant Characteristics}

Table 1 presents characteristics of participants with plausible habitual dietary intake who completed the total Flower FFQ and who did not complete the total Flower FFQ. For participants who completed the total Flower FFQ, the median (25th-75th percentile) age was 47 (39-56) years for men and $46(38-54)$ years for women. The majority of the participants were of white or east/west European ethnicity (98\% for men, $97 \%$ for women). The distribution over the northern three provinces and over the three SES categories was approximately equal. The median (25th-75th percentile) MVPA was 285 (120-627) minutes per week for men and 245 (115-520) minutes per week for women, and the median (25th-75th percentile) BMI was 25.9 (23.9-28.2) kg/m² for men and $24.8(22.5-27.9) \mathrm{kg} / \mathrm{m}^{2}$ for women.

Compared to participants who did not complete the total Flower FFQ, the median age of participants who completed the total Flower FFQ was a little higher. Accordingly, for completers, the percentage of participants was lower in the 18-50 years age category and higher in the 51-70 years age category compared to non-completers. However, in both groups, most participants were classified in the 18-50 years age group. In general, completers had a higher SES compared to non-completers, and this difference was more pronounced in men than in women. Among completers, less current smokers but more former smokers were present than among non-completers, and completers were slightly more physically active than non-completers. The distribution of participants over the northern three provinces of the Netherlands, and the median BMI was comparable between the two groups. Data on ethnicity were missing for a large number of participants who did not complete the total Flower FFQ, and it is therefore hard to compare ethnicity between the two groups. 
Table 1. Characteristics of participants with plausible habitual dietary who completed the total Flower FFQ $(n=59,982)$ and who did not complete the total Flower FFQ $(n=68,891)$.

\begin{tabular}{|c|c|c|c|c|c|c|c|c|c|c|}
\hline & \multicolumn{4}{|c|}{ Men $(n=53,026)$} & & \multicolumn{4}{|c|}{ Women $(n=75,847)$} & \\
\hline & \multicolumn{2}{|c|}{$\begin{array}{c}\text { Completed } \\
\text { Total Flower FFQ }(n=23,703)\end{array}$} & \multicolumn{2}{|c|}{$\begin{array}{c}\text { Did Not Complete } \\
\text { Total Flower FFQ }(n=29,323)\end{array}$} & & \multicolumn{2}{|c|}{$\begin{array}{c}\text { Completed } \\
\text { Total Flower FFQ }(n=36,279)\end{array}$} & \multicolumn{2}{|c|}{$\begin{array}{c}\text { Did Not Complete } \\
\text { Total Flower FFQ }(n=39,568)\end{array}$} & \\
\hline & Median/n & $\begin{array}{l}\text { 25th-75th } \\
\text { Percentile/\% }\end{array}$ & Median/n & $\begin{array}{l}\text { 25th-75th } \\
\text { Percentile/\% }\end{array}$ & & Median/n & $\begin{array}{l}\text { 25th-75th } \\
\text { Percentile/\% }\end{array}$ & Median/n & $\begin{array}{l}\text { 25th-75th } \\
\text { Percentile/\% }\end{array}$ & \\
\hline Age (years) & 47 & $39-56$ & 44 & $34-51$ & & 46 & $38-54$ & 43 & $33-50$ & \\
\hline $\begin{array}{l}\text { Age category } \\
18-50 \text { years } \\
51-70 \text { years } \\
>70 \text { years }\end{array}$ & $\begin{array}{c}14,890 \\
8144 \\
669\end{array}$ & $\begin{array}{c}62.8 \\
34.3 \\
2.8\end{array}$ & $\begin{array}{c}21,550 \\
6738 \\
1035\end{array}$ & $\begin{array}{c}73.5 \\
23.0 \\
3.5\end{array}$ & $* * *$ & $\begin{array}{c}24,361 \\
11,185 \\
733 \\
\end{array}$ & $\begin{array}{c}67.1 \\
30.8 \\
2.0 \\
\end{array}$ & $\begin{array}{c}30,199 \\
8344 \\
1025 \\
\end{array}$ & $\begin{array}{c}76.3 \\
21.1 \\
2.6\end{array}$ & $\begin{array}{l}* * * \\
* * *\end{array}$ \\
\hline $\begin{array}{l}\text { Province } \\
\text { Friesland } \\
\text { Groningen } \\
\text { Drenthe } \\
\text { Other } \\
\text { Unknown }\end{array}$ & $\begin{array}{l}8743 \\
7461 \\
6991 \\
502 \\
6\end{array}$ & $\begin{array}{c}36.9 \\
31.5 \\
29.5 \\
2.1 \\
0.0\end{array}$ & $\begin{array}{c}10,847 \\
8679 \\
8820 \\
725 \\
252 \\
\end{array}$ & $\begin{array}{c}37.0 \\
29.6 \\
30.1 \\
2.5 \\
0.9\end{array}$ & $* * *$ & $\begin{array}{c}13,562 \\
11,246 \\
10,641 \\
812 \\
15 \\
\end{array}$ & $\begin{array}{c}37.4 \\
31.0 \\
29.3 \\
2.2 \\
0.0\end{array}$ & $\begin{array}{c}14,683 \\
11,555 \\
11,978 \\
1055 \\
297 \\
\end{array}$ & $\begin{array}{c}37.1 \\
29.2 \\
30.3 \\
2.7 \\
0.8 \\
\end{array}$ & $* * *$ \\
\hline $\begin{array}{l}\text { Ethnicity } \\
\text { White, East/West } \\
\text { European } \\
\text { Other } \\
\text { Unknown }\end{array}$ & $\begin{array}{l}308 \\
163\end{array}$ & $\begin{array}{l}1.3 \\
0.7\end{array}$ & $\begin{array}{c}20,286 \\
448 \\
8589\end{array}$ & $\begin{array}{c}69.2 \\
1.5 \\
29.3\end{array}$ & $* * *$ & $\begin{array}{c}35,167 \\
628 \\
484\end{array}$ & $\begin{array}{l}1.7 \\
1.3\end{array}$ & $\begin{array}{c}28,212 \\
789 \\
10,567\end{array}$ & $\begin{array}{c}71.3 \\
2.0 \\
26.7\end{array}$ & $* * *$ \\
\hline $\begin{array}{l}\text { SES } \\
\text { Low } \\
\text { Moderate } \\
\text { High } \\
\text { Unknown }\end{array}$ & $\begin{array}{c}6590 \\
8563 \\
8143 \\
407\end{array}$ & $\begin{array}{c}27.8 \\
36.1 \\
34.4 \\
1.7\end{array}$ & $\begin{array}{c}8515 \\
11,317 \\
8781 \\
710\end{array}$ & $\begin{array}{c}29.0 \\
38.6 \\
29.9 \\
2.4\end{array}$ & $* * *$ & $\begin{array}{c}10,500 \\
14,314 \\
10,861 \\
604\end{array}$ & $\begin{array}{c}28.9 \\
39.5 \\
29.9 \\
1.7\end{array}$ & $\begin{array}{c}11,475 \\
16,167 \\
11,047 \\
879\end{array}$ & $\begin{array}{c}29.0 \\
40.9 \\
27.9 \\
2.2\end{array}$ & $* * *$ \\
\hline $\begin{array}{l}\text { Smoking } \\
\text { Current smoker } \\
\text { Former smoker } \\
\text { Never smoker } \\
\text { Unknown }\end{array}$ & $\begin{array}{c}4468 \\
8722 \\
10,359 \\
154\end{array}$ & $\begin{array}{c}18.8 \\
36.8 \\
43.7 \\
0.6\end{array}$ & $\begin{array}{c}7435 \\
9198 \\
12,452 \\
238\end{array}$ & $\begin{array}{c}25.4 \\
31.4 \\
42.5 \\
0.8\end{array}$ & $* * *$ & $\begin{array}{c}5876 \\
2480 \\
17,681 \\
242\end{array}$ & $\begin{array}{c}16.2 \\
34.4 \\
48.7 \\
0.7\end{array}$ & $\begin{array}{c}8718 \\
11,737 \\
18,769 \\
344\end{array}$ & $\begin{array}{c}22.0 \\
29.7 \\
47.4 \\
0.9\end{array}$ & $* * *$ \\
\hline
\end{tabular}


Table 1. Cont.

\begin{tabular}{|c|c|c|c|c|c|c|c|c|c|c|}
\hline & \multicolumn{4}{|c|}{ Men $(n=53,026)$} & & \multicolumn{4}{|c|}{ Women $(n=75,847)$} & \\
\hline & \multicolumn{2}{|c|}{$\begin{array}{c}\text { Completed } \\
\text { Total Flower FFQ }(n=23,703)\end{array}$} & \multicolumn{2}{|c|}{$\begin{array}{c}\text { Did Not Complete } \\
\text { Total Flower FFQ }(n=29,323)\end{array}$} & & \multicolumn{2}{|c|}{$\begin{array}{c}\text { Completed } \\
\text { Total Flower FFQ }(n=36,279)\end{array}$} & \multicolumn{2}{|c|}{$\begin{array}{c}\text { Did Not Complete } \\
\text { Total Flower FFQ }(n=39,568) \\
\end{array}$} & \\
\hline & Median/n & $\begin{array}{l}\text { 25th-75th } \\
\text { Percentile/\% }\end{array}$ & Median/n & $\begin{array}{l}\text { 25th-75th } \\
\text { Percentile/\% }\end{array}$ & & Median/n & $\begin{array}{l}\text { 25th-75th } \\
\text { Percentile/\% }\end{array}$ & Median/n & $\begin{array}{c}\text { 25th-75th } \\
\text { Percentile/\% }\end{array}$ & \\
\hline $\begin{array}{l}\text { Physical activity: MVPA } \\
\text { (minutes per week) }\end{array}$ & 285 & $120-627$ & 280 & $90-630$ & & 245 & $115-520$ & 240 & $90-480$ & \\
\hline Physical activity category & & & & & $* *$ & & & & & $* * *$ \\
\hline MVPA performance & 19,562 & 82.5 & 23,179 & 79.0 & $* * *$ & 31,361 & 86.4 & 32,707 & 82.7 & $* * *$ \\
\hline No MVPA performance & 2246 & 9.5 & 3252 & 11.1 & & 2743 & 7.6 & 4036 & 10.2 & \\
\hline Unknown or unreliable & 1895 & 8.0 & 2892 & 9.9 & & 2175 & 6.0 & 2825 & 7.1 & \\
\hline $\mathrm{BMI}\left(\mathrm{kg} / \mathrm{m}^{2}\right)$ & 25.9 & $23.9-28.2$ & 26.0 & $23.9-28.5$ & & 24.8 & $22.5-27.9$ & 25.0 & $22.5-28.4$ & \\
\hline \multicolumn{11}{|l|}{ BMI category } \\
\hline Normal weight & 8875 & 37.4 & 10,868 & 37.1 & $* *$ & 18,608 & 51.3 & 19,595 & 49.5 & $* * *$ \\
\hline Overweight & 11,690 & 49.3 & 13,909 & 47.4 & $* * *$ & 12,198 & 33.6 & 13,048 & 33.0 & $* * *$ \\
\hline Obesity & 3138 & 13.2 & 4546 & & & 5472 & 15.1 & 6925 & 17.5 & \\
\hline Unknown & & & 15.5 & & & 1 & 0.0 & & & \\
\hline
\end{tabular}

SES, socioeconomic status; MVPA, moderate to vigorous physical activity; BMI, body mass index. Continuous data are presented as median (25th-75th percentile), categorical data are presented as $n(\%)$. ${ }^{*}$ Significant difference between participants who completed the total Flower FFQ and who did not complete the total Flower FFQ. ${ }^{* *} p<0.01, * * *<0.001$. 


\subsection{Energy and Nutrient Intake}

Daily energy and nutrient intake are presented in Table 2. Median energy intake was higher in men $(2368 \mathrm{kcal})$ than in women $(1848 \mathrm{kcal})$, as well as absolute macronutrient and micronutrient intake, except for vitamin $\mathrm{C}$, of which the intake was higher in women. In terms of energy percentage $(\mathrm{En} \%)$, macronutrient intake in men and women were comparable, except for alcohol, of which the intake was 2.2 En\% in men and $0.9 \mathrm{En} \%$ in women.

Table 3 presents daily energy and nutrient intake per age category. In general, median energy and macronutrient intake was highest in the lowest age category and decreased subsequently in the middle and highest age categories, both in men and women. A clear exception on the above concerns alcohol intake, which was highest in the middle age category, both in men and women. For micronutrient intake in general, no clear trend was observed with increasing age categories. In higher age categories, both in men and women, intake of retinol equivalents, vitamin $C$ and calcium was a little higher compared to lower age categories, and in women only, this was true for folate, folate equivalents and vitamin B12 intake. On the other hand, intake of vitamin B6 and vitamin E was a little lower in higher age categories compared to lower age categories, particularly in men.

Table 4 presents daily energy and nutrient intake per SES category. No striking differences in daily energy and nutrient intake were observed among different categories of SES, except for alcohol intake in women, of which the intake was lowest in the low SES category and increased subsequently in the moderate and high SES categories.

Table 5 presents daily energy and nutrient intake per BMI category. A striking observation is that intake of energy and macronutrients was highest in participants with a normal weight and decreased with increasing BMI categories, both in men and women. An exception also concerns alcohol intake here, which was comparable in different BMI categories in men, whereas in women, alcohol intake was lower among obese participants compared to overweight participants and participants with a normal weight. Regarding micronutrient intake, no striking differences were observed among different categories of BMI.

\subsection{Food Intake}

Daily food intake, categorised into food groups, is presented in Table 6. Intake of food groups was different for men and women. Striking differences were that men consumed more alcoholic beverages, bread, coffee, fat, oils and sauces, meat, potatoes, ready-made products, savoury snacks, soft drinks and sweets, whereas women consumed more fruits, tea, vegetables and water.

Table 7 presents daily food intake per age category. Both in men and women, intake of the following food groups decreased with increasing age categories: artificially sweetened beverages, bread, fat, oils and sauces, fruit juice, meat, pasta, ready-made products, rice, savoury snacks and soft drinks. On the other hand, intake of cheese, dairy, eggs, fruits, tea and vegetables increased with increasing age categories.

Table 8 presents daily food intake per SES category. Both in men and women, participants with a higher SES consumed more fruit juice, ready-made products, savoury snacks and vegetables, compared to participants with a lower SES. A difference observed in women only was that participants with a higher SES consumed more alcoholic beverages compared to participants with a lower SES.

Table 9 presents daily food intake per BMI category. A striking observation is that consumption of cake and cookies, fat, oils and sauces, savoury snacks, soft drinks and sweets was highest in participants with a normal weight and decreased with increasing BMI categories, whereas consumption of artificially sweetened beverages was lowest in participants with a normal weight and increased with increasing BMI categories, both in men and women. 
Table 2. Daily energy and nutrient intake, obtained from the Flower FFQ $(n=59,982)$.

\begin{tabular}{|c|c|c|c|c|c|}
\hline & \multicolumn{2}{|c|}{ Men $(n=23,703)$} & \multicolumn{2}{|c|}{ Women $(n=36,279)$} & \\
\hline & Median & 25th-75th Percentile & Median & 25th-75th Percentile & \\
\hline Energy (kcal) & 2368 & 1974-2812 & 1848 & 1551-2179 & $* * *$ \\
\hline Total carbohydrates (g) & 255 & $209-308$ & 203 & $167-242$ & $* * *$ \\
\hline$(\mathrm{En} \%)$ & 45.3 & $41.6-49.2$ & 45.6 & $41.9-49.4$ & $* * *$ \\
\hline Mono- and disaccharides (g) & 108 & 83-138 & 89 & 69-112 & $* * *$ \\
\hline Polysaccharides (g) & 144 & $117-175$ & 112 & $91-133$ & $* * *$ \\
\hline Total fat (g) & 95 & $76-120$ & 73 & $58-91$ & $* * *$ \\
\hline$(\mathrm{En} \%)$ & 36.7 & $32.5-41.5$ & 35.7 & $31.6-40.0$ & $* * *$ \\
\hline Saturated fatty acids (g) & 33 & $26-41$ & 26 & $20-32$ & $* * *$ \\
\hline Monounsaturated fatty acids (g) & 34 & $26-42$ & 25 & $20-32$ & $* * *$ \\
\hline Polyunsaturated fatty acids (g) & 20 & $15-27$ & 14 & $11-19$ & $* * *$ \\
\hline Eicosapentaenoic acid (EPA) (g) & 0.04 & $0.01-0.09$ & 0.04 & $0.01-0.09$ & $* * *$ \\
\hline Docosahexaenoic acid (DHA) (g) & 0.06 & $0.02-0.13$ & 0.06 & $0.02-0.12$ & $* * *$ \\
\hline Total protein (g) & 84 & $71-98$ & 71 & $60-81$ & $* * *$ \\
\hline$(\mathrm{En} \%)$ & 14.9 & $13.6-16.4$ & 15.8 & $14.3-17.4$ & $* * *$ \\
\hline Vegetable protein (g) & 37 & $30-44$ & 29 & $24-35$ & $* * *$ \\
\hline Animal protein $(\mathrm{g})$ & 47 & $38-56$ & 41 & $34-49$ & $* * *$ \\
\hline Alcohol (g) & 6.8 & $2.6-15.3$ & 2.6 & $0.4-6.9$ & $* * *$ \\
\hline$(\mathrm{En} \%)$ & 2.2 & $0.8-4.4$ & 0.9 & $0.1-2.8$ & $* * *$ \\
\hline Fibre (g) & 25 & $20-30$ & 21 & $17-25$ & $* * *$ \\
\hline$(\mathrm{En} \%)$ & 2.0 & $1.7-2.3$ & 2.2 & $1.9-2.5$ & $* * *$ \\
\hline Retinol equivalents $(\mu \mathrm{g})$ & 1146 & $868-1542$ & 960 & $744-1232$ & $* * *$ \\
\hline Vitamin B2 (mg) & 1.5 & $1.2-1.8$ & 1.3 & $1.1-1.6$ & $* * *$ \\
\hline Vitamin B6 (mg) & 1.5 & $1.3-1.8$ & 1.3 & $1.1-1.5$ & $* * *$ \\
\hline Folate (present in food by nature) $(\mu \mathrm{g})$ & 253 & $210-303$ & 229 & $190-272$ & *** \\
\hline Folate equivalents $(\mu \mathrm{g})$ & 261 & $214-317$ & 234 & $193-282$ & $* * *$ \\
\hline Vitamin B12 ( $\mu \mathrm{g})$ & 4.0 & $3.1-5.4$ & 3.4 & $2.6-4.5$ & $* * *$ \\
\hline Vitamin C (mg) & 92 & $66-123$ & 96 & $69-127$ & $* * *$ \\
\hline Vitamin E (mg) & 13 & $10-17$ & 11 & 9-14 & $* * *$ \\
\hline Calcium (mg) & 986 & $786-1236$ & 920 & $731-1134$ & $* * *$ \\
\hline
\end{tabular}


Table 3. Daily energy and macronutrient intake per age category, obtained from the Flower FFQ $(n=59,982)$

\begin{tabular}{|c|c|c|c|c|c|c|c|c|c|c|c|c|c|c|}
\hline & \multicolumn{6}{|c|}{ Men $(n=23,703)$} & & \multicolumn{6}{|c|}{ Women $(n=36,279)$} & \\
\hline & \multicolumn{2}{|c|}{$18-50$ Years $(n=14,890)$} & \multicolumn{2}{|c|}{$51-70$ Years $(n=8144)$} & \multicolumn{2}{|c|}{$>70$ Years $(n=669)$} & & \multicolumn{2}{|c|}{$18-50$ Years $(n=24,361)$} & \multicolumn{2}{|c|}{$51-70$ Years $(n=11,185)$} & \multicolumn{2}{|c|}{$>70$ Years $(n=733)$} & \\
\hline & Median & $\begin{array}{l}\text { 25th-75th } \\
\text { Percentile }\end{array}$ & Median & $\begin{array}{l}\text { 25th-75th } \\
\text { Percentile }\end{array}$ & Median & $\begin{array}{l}\text { 25th-75th } \\
\text { Percentile }\end{array}$ & & Median & $\begin{array}{l}\text { 25th-75th } \\
\text { Percentile }\end{array}$ & Median & $\begin{array}{l}\text { 25th-75th } \\
\text { Percentile }\end{array}$ & Median & $\begin{array}{l}\text { 25th-75th } \\
\text { Percentile }\end{array}$ & \\
\hline Total carbohydrates (g) & 271 & $224-324$ & 232 & $192-279$ & 213 & $178-251$ & $* * * a b c$ & 210 & $174-249$ & 190 & $157-226$ & 183 & $153-212$ & $* * * a b c$ \\
\hline$(\mathrm{En} \%)$ & 46.1 & $42.4-49.8$ & 44.1 & $40.3-48.0$ & 44.0 & $39.7-47.7$ & $* * * a b$ & 46.2 & $42.6-49.9$ & 44.4 & $40.5-48.1$ & 44.7 & $40.4-48.5$ & $* * * a b$ \\
\hline Mono- and disaccharides (g) & 113 & $87-145$ & 99 & $76-127$ & 99 & $79-124$ & ${ }^{* * *} \mathrm{ab}$ & 91 & $71-115$ & 85 & $67-106$ & 88 & $71-108$ & $* * * a c$ \\
\hline Total fat (g) & 99 & $79-123$ & 89 & $71-113$ & 82 & 64-105 & $* * * a b c$ & 75 & $59-93$ & 69 & $55-86$ & 65 & $51-81$ & $* * * a b c$ \\
\hline$(\mathrm{En} \%)$ & 36.7 & $32.6-41.3$ & 36.8 & $32.3-42.1$ & 36.2 & $32.0-41.6$ & - & 36.9 & $32.0-40.2$ & 35.1 & $30.9-39.7$ & 34.4 & $30.0-39.1$ & $* * * a b c$ \\
\hline Saturated fatty acids (g) & 34 & $27-42$ & 31 & $24-39$ & 29 & $23-37$ & $* * * a b c$ & 26 & $21-33$ & 25 & $20-31$ & 24 & $19-31$ & $* * * a b$ \\
\hline Monounsaturated fatty acids (g) & 35 & $28-44$ & 31 & $24-40$ & 28 & $22-36$ & $* * * a b c$ & 26 & $21-33$ & 24 & $19-30$ & 21 & $17-27$ & $* * * a b c$ \\
\hline Polyunsaturated fatty acids (g) & 21 & $16-27$ & 19 & $14-25$ & 17 & $13-24$ & $* * * a b c$ & 15 & $11-19$ & 14 & $10-18$ & 13 & $9-17$ & $* * * a b c$ \\
\hline Eicosapentaenoic acid (EPA) (g) & 0.04 & $0.01-0.08$ & 0.06 & $0.02-0.10$ & 0.05 & $0.03-0.09$ & $* * * a b$ & 0.03 & $0.01-0.08$ & 0.06 & $0.02-0.10$ & 0.05 & $0.01-0.09$ & $* * * a b c$ \\
\hline Docosahexaenoic acid (DHA) (g) & 0.05 & $0.02-0.11$ & 0.08 & $0.03-0.15$ & 0.08 & $0.04-0.13$ & $* * * a b$ & 0.05 & $0.01-0.11$ & 0.08 & $0.03-0.15$ & 0.05 & $0.01-0.09$ & $* * * a b c$ \\
\hline$(\mathrm{En} \%)$ & 14.7 & $13.3-16.1$ & 15.3 & $14.0-16.8$ & 15.3 & $13.9-16.5$ & $* * * a b$ & 15.5 & $14.0-17.1$ & 16.4 & $14.9-18.1$ & 16.1 & $14.6-17.8$ & $* * * a b c$ \\
\hline Vegetable protein (g) & 38 & $31-46$ & 34 & $28-41$ & 29 & $24-36$ & $* * * a b c$ & 29 & $24-35$ & 28 & $23-33$ & 25 & $21-30$ & $* * * a b c$ \\
\hline Animal protein $(\mathrm{g})$ & 47 & $39-57$ & 46 & $38-55$ & 45 & $37-52$ & $* * * a b c$ & 41 & $33-49$ & 42 & $35-51$ & 40 & $33-48$ & $* * * a c$ \\
\hline Alcohol (g) & 6.6 & $2.5-13.3$ & 8.5 & $2.8-16.8$ & 6.3 & $1.6-12.5$ & $* * * a b c$ & 2.5 & $0.3-6.7$ & 3.3 & $0.7-9.3$ & 1.4 & $0.0-6.4$ & $* * * a b c$ \\
\hline$(E n \%)$ & 2 & $0.7-4.1$ & 2.7 & $1.0-5.1$ & 2.1 & $0.6-4.6$ & $* * * a c$ & 0.9 & $0.1-2.5$ & 1.4 & $0.2-3.7$ & 0.6 & $0.0-2.7$ & $* * * a b c$ \\
\hline Fibre $(\mathrm{g})$ & 25 & $21-31$ & 24 & $19-29$ & 22 & $18-26$ & $* * * a b c$ & 21 & $17-25$ & 21 & $18-25$ & 20 & $17-24$ & $* * * a b c$ \\
\hline$(\mathrm{En} \%)$ & 2 & $1.7-2.3$ & 2.1 & $1.8-2.4$ & 2.1 & $1.8-2.4$ & $* * * a b$ & 2.1 & $1.8-2.4$ & 2.3 & $2.0-2.6$ & 2.3 & $2.0-2.6$ & $* * * a b$ \\
\hline Retinol equivalents $(\mu \mathrm{g})$ & 1126 & 853-1504 & 1182 & 894-1597 & 1181 & $902-1612$ & $* * * a b$ & 941 & $724-1197$ & 1002 & 785-1301 & 995 & $788-1295$ & ${ }^{* * * a b}$ \\
\hline Vitamin B2 (mg) & 1.5 & $1.2-1.8$ & 1.5 & $1.2-1.8$ & 1.4 & $1.2-1.6$ & $* * * a b c$ & 1.3 & $1.0-1.5$ & 1.3 & $1.1-1.6$ & 1.3 & $1.1-1.6$ & $* * * a b$ \\
\hline Vitamin B6 (mg) & 1.6 & $1.3-1.8$ & 1.4 & $1.2-1.7$ & 1.3 & $1.1-1.6$ & $* * * a b c$ & 1.3 & $1.1-1.6$ & 1.3 & $1.1-1.5$ & 1.2 & $1.0-1.5$ & $* * * a b c$ \\
\hline
\end{tabular}


Table 3. Cont.

\begin{tabular}{|c|c|c|c|c|c|c|c|c|c|c|c|c|c|c|}
\hline & \multicolumn{6}{|c|}{ Men $(n=23,703)$} & & \multicolumn{6}{|c|}{ Women $(n=36,279)$} & \\
\hline & \multicolumn{2}{|c|}{$18-50$ Years $(n=14,890)$} & \multicolumn{2}{|c|}{$51-70$ Years $(n=8144)$} & \multicolumn{2}{|c|}{$>70$ Years $(n=669)$} & & \multicolumn{2}{|c|}{$18-50$ Years $(n=24,361)$} & \multicolumn{2}{|c|}{$51-70$ Years $(n=11,185)$} & \multicolumn{2}{|c|}{$>70$ Years $(n=733)$} & \\
\hline & Median & $\begin{array}{l}\text { 25th-75th } \\
\text { Percentile }\end{array}$ & Median & $\begin{array}{l}\text { 25th-75th } \\
\text { Percentile }\end{array}$ & Median & $\begin{array}{l}\text { 25th-75th } \\
\text { Percentile }\end{array}$ & & Median & $\begin{array}{l}\text { 25th-75th } \\
\text { Percentile }\end{array}$ & Median & $\begin{array}{l}\text { 25th-75th } \\
\text { Percentile }\end{array}$ & Median & $\begin{array}{l}\text { 25th-75th } \\
\text { Percentile }\end{array}$ & \\
\hline Folate equivalents $(\mu \mathrm{g})$ & 260 & $213-315$ & 263 & $216-322$ & 253 & $216-308$ & $* * * a$ & 227 & $188-273$ & 247 & $206-299$ & 246 & $206-302$ & $* * * a b$ \\
\hline Vitamin B12 $(\mu \mathrm{g})$ & 3.9 & $3.0-5.2$ & 4.1 & $3.2-5.6$ & 4.0 & $3.2-5.5$ & $* * * a$ & 3.3 & $2.5-4.3$ & 3.6 & $2.8-4.9$ & 3.5 & $2.7-4.6$ & $* * * a b$ \\
\hline Vitamin E (mg) & 14 & $11-18$ & 13 & $10-17$ & 12 & $9-16$ & $* * * a b c$ & 11 & $9-14$ & 11 & $9-14$ & 10 & $108-13$ & $* * * a b c$ \\
\hline Calcium (mg) & 978 & $775-1231$ & 1001 & $798-1247$ & 1003 & $817-1211$ & $* * * a$ & 890 & $707-1100$ & 974 & 788-1199 & 994 & 814-1196 & $* * * a b$ \\
\hline
\end{tabular}

${ }^{*}$ Significant difference between categories of age. ${ }^{*} p<0.05,{ }^{* * *} p<0.001$. a: significant difference between $18-50$ and $51-70$ years, b: significant difference between $18-50$ and $>70$ years, $\mathrm{c}$ significant difference between $51-70$ and $>70$ years.

Table 4. Daily energy and macronutrient intake per known SES category, obtained from the Flower FFQ $(n=58,971)$.

\begin{tabular}{|c|c|c|c|c|c|c|c|c|c|c|c|c|c|c|}
\hline & \multicolumn{6}{|c|}{ Men $(n=23,296)$} & & \multicolumn{6}{|c|}{ Women $(n=35,675)$} & \\
\hline & \multicolumn{2}{|c|}{ Low SES $(n=6590)$} & \multicolumn{2}{|c|}{$\begin{array}{c}\text { Moderate SES } \\
(n=8563)\end{array}$} & \multicolumn{2}{|c|}{ High SES $(n=8143)$} & & \multicolumn{2}{|c|}{ Low SES $(n=10,500)$} & \multicolumn{2}{|c|}{$\begin{array}{c}\text { Moderate SES } \\
(n=14,314)\end{array}$} & \multicolumn{2}{|c|}{ High SES $(n=10,861)$} & \\
\hline & Median & $\begin{array}{l}\text { 25th-75th } \\
\text { Percentile }\end{array}$ & Median & $\begin{array}{l}\text { 25th-75th } \\
\text { Percentile }\end{array}$ & Median & $\begin{array}{l}\text { 25th-75th } \\
\text { Percentile }\end{array}$ & & Median & $\begin{array}{l}\text { 25th-75th } \\
\text { Percentile }\end{array}$ & Median & $\begin{array}{l}\text { 25th-75th } \\
\text { Percentile }\end{array}$ & Median & $\begin{array}{l}\text { 25th-75th } \\
\text { Percentile }\end{array}$ & \\
\hline Energy (kcal) & 2355 & 1957-2834 & 2432 & 2013-2892 & 2319 & 1949-2725 & $* * * a b c$ & 1801 & $1512-2135$ & 1868 & 1564-2199 & 1868 & $1576-2188$ & $* * * a b$ \\
\hline Total carbohydrates (g) & 253 & $207-309$ & 264 & $214-317$ & 249 & 206-299 & $* * * a b c$ & 195 & $161-235$ & 206 & $170-245$ & 206 & $170-244$ & $* * * a b$ \\
\hline$(\mathrm{En} \%)$ & 45.1 & $41.2-49.0$ & 45.5 & $41.8-49.3$ & 45.4 & $41.8-49.1$ & ${ }^{* * *} \mathrm{ab}$ & 45 & $41.1-48.9$ & 45.8 & $42.1-49.5$ & 45.9 & $42.4-49.6$ & $* * * a b c$ \\
\hline Polysaccharides (g) & 141 & 114-174 & 148 & $120-180$ & 143 & $118-173$ & $* * * a c$ & 106 & $87-128$ & 113 & $93-135$ & 115 & $94-137$ & $* * * a b c$ \\
\hline Total fat $(\mathrm{g})$ & 96 & $75-122$ & 98 & $77-124$ & 93 & $74-114$ & $* * * a b c$ & 72 & $56-90$ & 74 & $58-93$ & 73 & $58-90$ & $* * * a b c$ \\
\hline$(\mathrm{En} \%)$ & 36.9 & $32.3-42.2$ & 36.9 & $32.5-41.8$ & 36.5 & $32.6-40.8$ & $* * * \mathrm{bc}$ & 35.6 & $31.2-40.3$ & 35.9 & $31.9-40.3$ & 35.5 & $31.7-39.5$ & $* * * a c$ \\
\hline Saturated fatty acids (g) & 33 & $26-41$ & 33 & $26-42$ & 32 & $26-40$ & $* * * a b c$ & 25 & $20-32$ & 26 & $21-33$ & 26 & $21-32$ & $* * * a b$ \\
\hline Monounsaturated fatty acids (g) & 33 & $26-43$ & 35 & $27-44$ & 33 & $26-41$ & $* * * a b c$ & 25 & $19-31$ & 26 & $20-33$ & 25 & $20-32$ & $* * * a b c$ \\
\hline
\end{tabular}


Table 4. Cont.

\begin{tabular}{|c|c|c|c|c|c|c|c|c|c|c|c|c|c|c|}
\hline & \multicolumn{6}{|c|}{ Men $(n=23,296)$} & & \multicolumn{6}{|c|}{ Women $(n=35,675)$} & \\
\hline & \multicolumn{2}{|c|}{ Low SES $(n=6590)$} & \multicolumn{2}{|c|}{$\begin{array}{l}\text { Moderate SES } \\
\quad(n=8563)\end{array}$} & \multicolumn{2}{|c|}{ High SES $(n=8143)$} & & \multicolumn{2}{|c|}{ Low SES $(n=10,500)$} & \multicolumn{2}{|c|}{$\begin{array}{c}\text { Moderate SES } \\
(n=14,314)\end{array}$} & \multicolumn{2}{|c|}{ High SES $(n=10,861)$} & \\
\hline & Median & $\begin{array}{l}\text { 25th-75th } \\
\text { Percentile }\end{array}$ & Median & $\begin{array}{l}\text { 25th-75th } \\
\text { Percentile }\end{array}$ & Median & $\begin{array}{l}\text { 25th-75th } \\
\text { Percentile }\end{array}$ & & Median & $\begin{array}{l}\text { 25th-75th } \\
\text { Percentile }\end{array}$ & Median & $\begin{array}{l}\text { 25th-75th } \\
\text { Percentile }\end{array}$ & Median & $\begin{array}{l}\text { 25th-75th } \\
\text { Percentile }\end{array}$ & \\
\hline Polyunsaturated fatty acids (g) & 21 & $15-28$ & 21 & $15-27$ & 19 & $14-25$ & $* * * \mathrm{bc}$ & 15 & $11-19$ & 15 & $11-19$ & 14 & $11-18$ & $* * * b c$ \\
\hline Eicosapentaenoic acid (EPA) (g) & 0.04 & $0.01-0.08$ & 0.04 & $0.01-0.08$ & 0.06 & $0.02-0.10$ & $* * * \mathrm{bc}$ & 0.04 & $0.01-0.08$ & 0.04 & $0.01-0.08$ & 0.05 & $0.02-0.10$ & ${ }^{* * *} \mathrm{bc}$ \\
\hline Docosahexaenoic acid (DHA) (g) & 0.06 & $0.02-0.11$ & 0.05 & $0.02-0.11$ & 0.08 & $0.03-0.15$ & $* * * \mathrm{bc}$ & 0.05 & $0.01-0.11$ & 0.05 & $0.01-0.11$ & 0.08 & $0.02-0.14$ & $* *$ bc \\
\hline$(\mathrm{En} \%)$ & 14.6 & $13.3-16.1$ & 14.7 & $13.4-16.2$ & 15.3 & $14.0-16.7$ & $* *$ abc & 15.8 & $14.2-17.5$ & 15.6 & $14.1-17.3$ & 16 & $14.5-17.6$ & $* * * a b c$ \\
\hline Vegetable protein $(\mathrm{g})$ & 35 & $28-43$ & 37 & $30-45$ & 37 & $30-44$ & $* * * a b$ & 27 & $23-33$ & 29 & $24-34$ & 30 & $25-36$ & $* * * a b c$ \\
\hline Animal protein $(\mathrm{g})$ & 46 & $38-56$ & 47 & $39-57$ & 47 & $38-55$ & $* * * a c$ & 41 & $33-49$ & 41 & $34-49$ & 41 & $33-49$ & ${ }^{*} \mathrm{C}$ \\
\hline Alcohol (g) & 6.7 & $2.5-15.4$ & 6.7 & $2.5-15.0$ & 6.8 & $2.7-15.5$ & $* * b$ & 2 & $0.0-6.9$ & 2.5 & $0.3-6.7$ & 3.4 & $0.9-8.3$ & $* * * a b c$ \\
\hline$(\mathrm{En} \%)$ & 2.2 & $0.7-4.4$ & 2.1 & $0.8-4.3$ & 2.4 & $0.9-4.5$ & $* * * \mathrm{bc}$ & 0.8 & $0.0-2.8$ & 0.9 & $0.1-2.6$ & 1.3 & $0.4-3.2$ & $* * * a b c$ \\
\hline Fibre $(\mathrm{g})$ & 24 & $19-29$ & 25 & $20-30$ & 25 & $21-30$ & $* * * a b$ & 20 & $17-24$ & 21 & $17-25$ & 22 & $18-26$ & $* *$ abc \\
\hline$(\mathrm{En} \%)$ & 2 & $1.7-2.3$ & 2 & $1.7-2.3$ & 2.1 & $1.8-2.4$ & $* * * \mathrm{bc}$ & 2.1 & $1.8-2.5$ & 2.1 & $1.8-2.5$ & 2.3 & $2.0-2.6$ & $* * * a b c$ \\
\hline Vitamin B2 (mg) & 1.5 & $1.2-1.8$ & 1.5 & $1.2-1.8$ & 1.5 & $1.2-1.8$ & $* * *$ ac & 1.3 & $1.1-1.6$ & 1.3 & $1.0-1.6$ & 1.3 & $1.1-1.6$ & - \\
\hline Vitamin B6 (mg) & 1.5 & $1.2-1.8$ & 1.3 & $1.3-1.8$ & 1.5 & $1.3-1.8$ & $* * * a b c$ & 1.3 & $1.1-1.5$ & 1.3 & $1.1-1.5$ & 1.4 & $1.2-1.6$ & $* * * a b c$ \\
\hline $\begin{array}{l}\text { Folate } \\
\text { (present in food by nature) }(\mu \mathrm{g})\end{array}$ & 250 & 203-299 & 252 & $209-303$ & 258 & 215-305 & $* * * a b c$ & 222 & 184-265 & 224 & $186-265$ & 241 & 203-286 & $* * * \mathrm{bc}$ \\
\hline Folate equivalents $(\mu \mathrm{g})$ & 256 & 208-312 & 259 & $213-316$ & 267 & $221-321$ & $* * * a b c$ & 227 & $187-276$ & 228 & 189-274 & 247 & $207-297$ & $* * * \mathrm{bc}$ \\
\hline Vitamin B12 ( $\mu \mathrm{g})$ & 4.0 & $3.0-5.4$ & 4.0 & $3.0-5.3$ & 4.1 & $3.1-5.5$ & $* * * \mathrm{bc}$ & 3.4 & $2.6-4.5$ & 3.3 & $2.6-4.4$ & 3.5 & $2.7-4.7$ & $* * * a b c$ \\
\hline Vitamin C (mg) & 88 & $62-120$ & 90 & $65-122$ & 96 & $71-127$ & $* * * a b c$ & 96 & $68-127$ & 92 & $66-123$ & 101 & 74-131 & $* * * a b c$ \\
\hline Vitamin E (mg) & 13 & $10-17$ & 14 & $11-18$ & 13 & $10-17$ & ${ }^{* * *} \mathrm{bc}$ & 11 & $9-14$ & 11 & $9-14$ & 11 & $9-14$ & ${ }^{* * a c}$ \\
\hline Calcium (mg) & 977 & $775-1232$ & 987 & $785-1241$ & 993 & $792-1233$ & - & 925 & $735-1137$ & 908 & $722-1121$ & 929 & $743-1142$ & $* * * a c$ \\
\hline
\end{tabular}

high SES, c: significant difference between moderate and high SES. 
Table 5. Daily energy and macronutrient intake per known BMI category, obtained from the Flower FFQ $(n=59,981)$.

\begin{tabular}{|c|c|c|c|c|c|c|c|c|c|c|c|c|c|c|}
\hline & \multicolumn{6}{|c|}{ Men $(n=23,703)$} & & \multicolumn{6}{|c|}{ Women $(n=36,278)$} & \\
\hline & \multicolumn{2}{|c|}{$\begin{array}{l}\text { Normal Weight } \\
\quad(n=8875)\end{array}$} & \multicolumn{2}{|c|}{$\begin{array}{l}\text { Overweight } \\
(n=11,690)\end{array}$} & \multicolumn{2}{|c|}{ Obesity $(n=3138)$} & & \multicolumn{2}{|c|}{$\begin{array}{l}\text { Normal Weight } \\
\quad(n=18,608)\end{array}$} & \multicolumn{2}{|c|}{$\begin{array}{l}\text { Overweight } \\
(n=12,198)\end{array}$} & \multicolumn{2}{|c|}{ Obesity $(n=5472)$} & \\
\hline & Median & $\begin{array}{l}\text { 25th-75th } \\
\text { Percentile }\end{array}$ & Median & $\begin{array}{l}\text { 25th-75th } \\
\text { Percentile }\end{array}$ & Median & $\begin{array}{l}\text { 25th-75th } \\
\text { Percentile }\end{array}$ & & Median & $\begin{array}{l}\text { 25th-75th } \\
\text { Percentile }\end{array}$ & Median & $\begin{array}{l}\text { 25th-75th } \\
\text { Percentile }\end{array}$ & Median & $\begin{array}{l}\text { 25th-75th } \\
\text { Percentile }\end{array}$ & \\
\hline Energy (kcal) & 2473 & 2081-2910 & 2324 & $1937-2765$ & 2218 & $1823-2689$ & $* * * a b c$ & 1898 & 1602-2219 & 1806 & 1519-2139 & 1766 & $1461-2100$ & ***abc \\
\hline Total carbohydrates (g) & 272 & $226-326$ & 249 & $204-300$ & 232 & 188-283 & $* * * a b c$ & 210 & $175-248$ & 196 & $161-234$ & 192 & $155-233$ & ${ }^{* * * a b c}$ \\
\hline$(E n \%)$ & 46.3 & $42.8-50.1$ & 45 & $41.3-48.7$ & 43.8 & $39.5-47.8$ & $* *$ abc & 46.2 & $42.6-49.9$ & 45.2 & $41.3-48.8$ & 44.8 & $40.8-48.5$ & $* * * b c$ \\
\hline Polysaccharides (g) & 153 & $126-184$ & 141 & $114-170$ & 134 & 108-165 & $* * * a b c$ & 115 & $95-137$ & 108 & $88-130$ & 107 & $86-130$ & $* * * a b$ \\
\hline Total fat $(\mathrm{g})$ & 98 & $79-122$ & 94 & $74-118$ & 92 & $71-116$ & $* * * a b c$ & 75 & $59-93$ & 71 & $57-90$ & 70 & $55-88$ & ${ }^{* * * a b c}$ \\
\hline$(\mathrm{En} \%)$ & 36.5 & $32.3-41.1$ & 36.8 & $32.5-41.6$ & 37 & $32.7-42.2$ & $* * * a b$ & 35.7 & $31.7-40.1$ & 35.7 & $31.6-40.0$ & 35.4 & $31.4-40.0$ & - \\
\hline Saturated fatty acids (g) & 34 & $27-42$ & 32 & $26-40$ & 31 & $24-40$ & $* *$ abc & 27 & $21-33$ & 25 & $20-32$ & 25 & $19-31$ & $* * * a b c$ \\
\hline Monounsaturated fatty acids (g) & 35 & $28-43$ & 33 & $26-42$ & 32 & $25-42$ & $* * * a b c$ & 26 & $20-33$ & 25 & $20-31$ & 25 & $19-31$ & ${ }^{* * *} \mathrm{abc}$ \\
\hline Polyunsaturated fatty acids (g) & 20 & $16-27$ & 20 & $15-26$ & 19 & $14-26$ & $* * * a b c$ & 15 & $11-19$ & 14 & $11-19$ & 14 & $10-18$ & ***abc \\
\hline Eicosapentaenoic acid (EPA) (g) & 0.04 & $0.01-0.09$ & 0.05 & $0.02-0.09$ & 0.05 & $0.02-0.09$ & $* * * a b$ & 0.04 & $0.01-0.09$ & 0.04 & $0.01-0.09$ & 0.04 & $0.01-0.08$ & ${ }^{* * * * b c}$ \\
\hline Total protein $(\mathrm{g})$ & 86 & 73-99 & 83 & 71-97 & 82 & 69-97 & $* * * a b$ & 71 & $60-82$ & 70 & 60-81 & 70 & 59-81 & $*$ \\
\hline$(\mathrm{En} \%)$ & 14.6 & $13.3-16.0$ & 15 & $13.7-16.5$ & 15.5 & 13.9-17.0 & $* * * a b c$ & 15.5 & $14.0-17.1$ & 16 & $14.5-17.7$ & 16.2 & $14.6-18.0$ & ${ }^{* * *} \mathrm{abc}$ \\
\hline Vegetable protein $(\mathrm{g})$ & 39 & $32-47$ & 36 & $29-43$ & 34 & $27-42$ & $* * * a b c$ & 30 & $25-35$ & 28 & $23-34$ & 27 & $22-33$ & $* * a b c$ \\
\hline Animal protein $(\mathrm{g})$ & 46 & $38-55$ & 47 & $39-56$ & 48 & $39-58$ & $* * * a b c$ & 40 & $33-48$ & 42 & $34-50$ & 42 & $35-50$ & $* * * a b$ \\
\hline Alcohol (g) & 6.7 & $2.6-13.3$ & 6.9 & $2.6-15.9$ & 6.7 & $1.9-15.8$ & $* * * a c$ & 2.9 & $0.7-7.1$ & 2.6 & $0.3-7.1$ & 1.3 & $0.0-5.2$ & $* * * a b c$ \\
\hline$(E n \%)$ & 2.1 & $0.7-4.1$ & 2.4 & $0.9-4.7$ & 2.2 & $0.6-4.8$ & $* * * a b c$ & 1.1 & $0.3-2.9$ & 1 & $0.1-3.0$ & 0.5 & $0.0-1.9$ & ${ }^{* * * a b c}$ \\
\hline Fibre $(\mathrm{g})$ & 26 & $21-31$ & 24 & $20-29$ & 23 & $19-28$ & $* *$ abc & 21 & $18-26$ & 21 & $17-25$ & 20 & $16-24$ & ${ }^{* * * a b c}$ \\
\hline$(\mathrm{En} \%)$ & 2 & $1.7-2.3$ & 2 & $1.7-2.3$ & 2 & $1.7-2.3$ & $* * * a b$ & 2.2 & $1.9-2.5$ & 2.2 & $1.9-2.5$ & 2.2 & $1.9-2.5$ & - \\
\hline Retinol equivalents $(\mu \mathrm{g})$ & 1151 & 879-1519 & 1142 & $865-1548$ & 1155 & 844-1589 & - & 955 & $745-1221$ & 963 & $745-1233$ & 964 & $740-1273$ & *b \\
\hline Vitamin B2 (mg) & 1.5 & $1.2-1.8$ & 1.5 & $1.2-1.8$ & 1.4 & $1.2-1.8$ & $* * * \mathrm{bc}$ & 1.3 & $1.0-1.6$ & 1.3 & $1.1-1.6$ & 1.3 & $1.0-1.6$ & $* a$ \\
\hline Vitamin B6 (mg) & 1.5 & $1.3-1.8$ & 1.5 & $1.2-1.8$ & 1.4 & $1.2-1.7$ & $* * * a b c$ & 1.3 & $1.1-1.5$ & 1.3 & $1.1-1.5$ & 1.3 & $1.1-1.5$ & ${ }^{* * * a b c}$ \\
\hline
\end{tabular}


Table 5. Cont.

\begin{tabular}{|c|c|c|c|c|c|c|c|c|c|c|c|c|c|c|}
\hline & \multicolumn{6}{|c|}{ Men $(n=23,703)$} & & \multicolumn{6}{|c|}{ Women $(n=36,278)$} & \\
\hline & \multicolumn{2}{|c|}{$\begin{array}{l}\text { Normal Weight } \\
\quad(n=8875)\end{array}$} & \multicolumn{2}{|c|}{$\begin{array}{l}\text { Overweight } \\
(n=11,690)\end{array}$} & \multicolumn{2}{|c|}{ Obesity $(n=3138)$} & & \multicolumn{2}{|c|}{$\begin{array}{l}\text { Normal Weight } \\
\quad(n=18,608)\end{array}$} & \multicolumn{2}{|c|}{$\begin{array}{l}\text { Overweight } \\
(n=12,198)\end{array}$} & \multicolumn{2}{|c|}{ Obesity $(n=5472)$} & \\
\hline & Median & $\begin{array}{l}\text { 25th-75th } \\
\text { Percentile }\end{array}$ & Median & $\begin{array}{l}\text { 25th-75th } \\
\text { Percentile }\end{array}$ & Median & $\begin{array}{l}\text { 25th-75th } \\
\text { Percentile }\end{array}$ & & Median & $\begin{array}{l}\text { 25th-75th } \\
\text { Percentile }\end{array}$ & Median & $\begin{array}{l}\text { 25th-75th } \\
\text { Percentile }\end{array}$ & Median & $\begin{array}{l}\text { 25th-75th } \\
\text { Percentile }\end{array}$ & \\
\hline $\begin{array}{l}\text { Folate } \\
\text { (present in food by nature) }(\mu \mathrm{g})\end{array}$ & 261 & $217-313$ & 250 & $207-298$ & 243 & 200-293 & $* * * a b c$ & 231 & $192-275$ & 227 & $189-270$ & 223 & $183-265$ & ***abc \\
\hline Folate equivalents ( $\mu \mathrm{g}$ ) & 269 & $222-327$ & 258 & $212-312$ & 248 & 204-303 & $* * * a b c$ & 236 & 195-285 & 233 & 193-280 & 227 & $86-273$ & ***abc \\
\hline Vitamin B12 ( $\mu \mathrm{g})$ & 3.9 & $3.0-5.1$ & 4.1 & $3.1-5.4$ & 4.2 & $3.1-5.8$ & $* * * a b c$ & 3.4 & $2.6-4.4$ & 3.4 & $2.7-4.6$ & 3.5 & $2.7-4.7$ & $* * * a b$ \\
\hline Vitamin C (mg) & 93 & $67-125$ & 91 & $66-122$ & 88 & $63-120$ & $* * * a b c$ & 96 & $70-127$ & 97 & $70-127$ & 94 & $66-124$ & $* *$ bc \\
\hline Vitamin E (mg) & 14 & $11-17$ & 13 & $10-17$ & 13 & $10-17$ & $* * * a b c$ & 11 & $9-14$ & 11 & $9-14$ & 11 & $8-14$ & $* * * a b$ \\
\hline Calcium (mg) & 998 & $797-1250$ & 986 & $786-1232$ & 957 & $746-1205$ & $* * * \mathrm{bc}$ & 920 & $728-1135$ & 924 & $739-1140$ & 907 & $726-1120$ & ${ }^{* *} \mathrm{C}$ \\
\hline
\end{tabular}

* Significant difference between categories of BMI. ${ }^{*} p<0.05,{ }^{* *} p<0.01,{ }^{* * *} p<0.001$. a: significant difference between normal weight and overweight, b: significant difference between normal weight and obesity, c: significant difference between overweight and obesity. 
Table 6. Daily food intake, categorised into food groups, obtained from the Flower FFQ $(n=59,982)$.

\begin{tabular}{|c|c|c|c|c|c|}
\hline & \multicolumn{2}{|c|}{ Men $(n=23,703)$} & \multicolumn{2}{|c|}{ Women $(n=36,279)$} & \\
\hline & Median & $\begin{array}{l}\text { 25th-75th } \\
\text { Percentile }\end{array}$ & Median & $\begin{array}{l}\text { 25th-75th } \\
\text { Percentile }\end{array}$ & \\
\hline Alcoholic beverages (g) & 107 & $36-215$ & 29 & $4-85$ & *** \\
\hline Artificially sweetened beverages (g) & 9 & $0-80$ & 13 & $0-80$ & *** \\
\hline Bread $(\mathrm{g})$ & 163 & $120-208$ & 116 & $83-148$ & $* * *$ \\
\hline Breakfast cereals (g) & 0 & $0-6$ & 0 & $0-8$ & $* * *$ \\
\hline Cake and cookies $(\mathrm{g})$ & 30 & $18-48$ & 30 & $18-46$ & ** \\
\hline Cheese (g) & 26 & $14-43$ & 23 & $13-40$ & $* * *$ \\
\hline Coffee $(\mathrm{g})$ & 465 & $348-697$ & 348 & $161-465$ & *** \\
\hline Dairy (g) & 291 & $180-427$ & 282 & $170-413$ & *** \\
\hline Eggs (g) & 9 & $7-18$ & 7 & $4-18$ & $* * *$ \\
\hline Fat, oils and sauces (g) & 57 & $37-81$ & 40 & $26-58$ & $* * *$ \\
\hline Fish (g) & 13 & $4-22$ & 12 & $3-20$ & $* * *$ \\
\hline Fruits (g) & 105 & $44-203$ & 133 & $68-205$ & $* * *$ \\
\hline Fruit juice (g) & 21 & $0-96$ & 21 & $0-54$ & $* * *$ \\
\hline Legumes (g) & 7 & $0-16$ & 4 & $0-11$ & $* * *$ \\
\hline Meat (g) & 86 & 64-109 & 70 & $46-93$ & $* * *$ \\
\hline Nuts and seeds (g) & 10 & $5-21$ & 7 & $3-14$ & $* * *$ \\
\hline Pasta (g) & 19 & $12-32$ & 19 & $12-26$ & $* * *$ \\
\hline Potatoes $(\mathrm{g})$ & 95 & 53-138 & 71 & 40-104 & $* * *$ \\
\hline $\begin{array}{l}\text { Probiotics and drinks lowering } \\
\text { cholesterol and blood pressure (g) }\end{array}$ & 0 & $0-0$ & 0 & $0-0$ & ** \\
\hline Ready-made products (g) & 31 & $6-51$ & 18 & $1-36$ & $* * *$ \\
\hline Rice $(\mathrm{g})$ & 20 & $8-32$ & 16 & $6-25$ & *** \\
\hline Savoury snacks (g) & 32 & $16-51$ & 22 & $11-39$ & $* * *$ \\
\hline Soft drinks (g) & 34 & $0-116$ & 9 & $0-62$ & *** \\
\hline Soup $(g)$ & 36 & $22-89$ & 36 & $22-72$ & *** \\
\hline Soy products $(\mathrm{g})$ & 0 & $0-0$ & 0 & $0-0$ & $* * *$ \\
\hline Sweets (g) & 34 & $17-57$ & 25 & $12-42$ & $* * *$ \\
\hline Tea $(\mathrm{g})$ & 116 & $11-232$ & 232 & $89-465$ & $* * *$ \\
\hline Vegan products other than soy (g) & 0 & $0-0$ & 0 & $0-0$ & $* * *$ \\
\hline Vegetables (g) & 131 & 89-184 & 148 & $106-205$ & *** \\
\hline Water $(\mathrm{g})$ & 279 & $107-482$ & 418 & $161-557$ & *** \\
\hline
\end{tabular}


Table 7. Daily food intake, categorised into food groups, per age category, obtained from the Flower FFQ $(n=59,982)$.

\begin{tabular}{|c|c|c|c|c|c|c|c|c|c|c|c|c|c|c|}
\hline & \multicolumn{6}{|c|}{ Men $(n=23,703)$} & & \multicolumn{6}{|c|}{ Women $(n=36,279)$} & \\
\hline & \multicolumn{2}{|c|}{$18-50$ Years $(n=14,890)$} & \multicolumn{2}{|c|}{ 51-70 Years $(n=8144)$} & \multicolumn{2}{|c|}{$>70$ Years $(n=669)$} & & \multicolumn{2}{|c|}{$18-50$ Years $(n=24,361)$} & \multicolumn{2}{|c|}{$51-70$ Years $(n=11,185)$} & \multicolumn{2}{|c|}{$>70$ Years $(n=733)$} & \\
\hline & Median & $\begin{array}{l}\text { 25th-75th } \\
\text { Percentile }\end{array}$ & Median & $\begin{array}{l}\text { 25th-75th } \\
\text { Percentile }\end{array}$ & Median & $\begin{array}{l}\text { 25th-75th } \\
\text { Percentile }\end{array}$ & & Median & $\begin{array}{l}\text { 25th-75th } \\
\text { Percentile }\end{array}$ & Median & $\begin{array}{l}\text { 25th-75th } \\
\text { Percentile }\end{array}$ & Median & $\begin{array}{l}\text { 25th-75th } \\
\text { Percentile }\end{array}$ & \\
\hline Artificially sweetened beverages (g) & 13 & $0-95$ & 5 & $0-54$ & 0 & $0-27$ & $* * * a b c$ & 18 & $0-96$ & 5 & $0-43$ & 0 & $0-21$ & ***abc \\
\hline Bread $(\mathrm{g})$ & 169 & $129-214$ & 149 & $111-198$ & 132 & $100-166$ & $* * * a b c$ & 119 & $84-151$ & 114 & $82-145$ & 108 & $78-137$ & $* * * a b c$ \\
\hline Breakfast cereals (g) & 0 & $0-6$ & 0 & $0-6$ & 0 & $0-6$ & $* * * \mathrm{a}$ & 0 & $0-9$ & 0 & $0-6$ & 0 & $0-6$ & $* * * a b$ \\
\hline Cheese (g) & 24 & $12-41$ & 29 & $17-46$ & 30 & $18-47$ & $* * * a b$ & 21 & $11-37$ & 28 & $17-43$ & 29 & $18-42$ & $* * * a b$ \\
\hline Coffee $(\mathrm{g})$ & 465 & $322-697$ & 465 & $348-697$ & 465 & $348-523$ & $* * * a b c$ & 348 & $45-465$ & 465 & $241-581$ & 348 & $232-465$ & $* * * a b c$ \\
\hline Dairy (g) & 290 & $177-430$ & 292 & $183-421$ & 307 & $204-422$ & - & 276 & $164-400$ & 293 & $183-422$ & 309 & $236-440$ & $* * * a b c$ \\
\hline Eggs (g) & 9 & $7-18$ & 14 & $7-18$ & 18 & $7-18$ & $* * * a b c$ & 7 & $4-18$ & 9 & $7-18$ & 18 & $7-18$ & $* * * a b c$ \\
\hline Fat, oils and sauces (g) & 59 & $40-84$ & 53 & $34-77$ & 47 & $30-70$ & $* * * a b c$ & 42 & $27-61$ & 36 & $23-53$ & 33 & $21-49$ & $* * * a b c$ \\
\hline Fish $(\mathrm{g})$ & 11 & $3-20$ & 16 & $8-25$ & 15 & $9-22$ & ${ }^{* * *} \mathrm{ab}$ & 11 & $1-19$ & 15 & $6-24$ & 13 & $5-20$ & $* * * a b c$ \\
\hline Fruits (g) & 89 & 40-192 & 130 & $69-211$ & 197 & $101-231$ & $* * * a b c$ & 104 & $48-199$ & 193 & $85-216$ & 205 & $141-292$ & $* * * a b c$ \\
\hline Legumes $(\mathrm{g})$ & 7 & $0-16$ & 9 & $0-18$ & 9 & $0-16$ & $* * * a b$ & 4 & $0-11$ & 7 & $0-16$ & 7 & $0-16$ & ${ }^{* * *} \mathrm{ab}$ \\
\hline Meat $(\mathrm{g})$ & 90 & $66-112$ & 80 & $61-103$ & 71 & $48-93$ & $* * * a b c$ & 72 & $49-95$ & 67 & $41-89$ & 62 & $36-75$ & $* * * a b c$ \\
\hline Nuts and seeds (g) & 11 & $5-21$ & 10 & $4-21$ & 6 & $3-14$ & $* * * b c$ & 7 & $3-14$ & 7 & $3-15$ & 4 & $1-10$ & $* * * b c$ \\
\hline Pasta (g) & 24 & 13-39 & 19 & $8-26$ & 12 & $3-18$ & $* * * a b c$ & 19 & $12-32$ & 13 & $8-19$ & 8 & $3-13$ & $* * * a b c$ \\
\hline Potatoes $(\mathrm{g})$ & 97 & $55-140$ & 90 & $50-135$ & 90 & $40-135$ & $* * * a b$ & 72 & $42-105$ & 65 & $34-104$ & 69 & $27-103$ & $* * * a b$ \\
\hline $\begin{array}{l}\text { Probiotics and drinks lowering } \\
\text { cholesterol and blood pressure (g) }\end{array}$ & 0 & $0-0$ & 0 & $0-0$ & 0 & $0-0$ & - & 0 & $0-0$ & 0 & $0-0$ & 0 & $0-0$ & $* a$ \\
\hline Ready-made products (g) & 33 & $13-53$ & 14 & $0-33$ & 1 & $0-26$ & $* * * a b c$ & 31 & $12-43$ & 13 & $0-32$ & 0 & $0-15$ & $* * * a b c$ \\
\hline Rice (g) & 20 & $10-34$ & 16 & $6-26$ & 10 & $0-20$ & $* * * a b c$ & 16 & $6-26$ & 15 & $4-24$ & 10 & $0-16$ & $* * * a b c$ \\
\hline Savoury snacks (g) & 39 & $22-59$ & 20 & $9-37$ & 8 & $2-17$ & $* * * a b c$ & 28 & $15-44$ & 14 & $5-25$ & 6 & $1-14$ & $* * * a b c$ \\
\hline Soft drinks (g) & 62 & $11-156$ & 10 & $0-52$ & 0 & $0-31$ & $* * * a b c$ & 21 & $0-94$ & 0 & $0-18$ & 0 & $0-13$ & $* * * a b$ \\
\hline
\end{tabular}


Table 7. Cont.

\begin{tabular}{|c|c|c|c|c|c|c|c|c|c|c|c|c|c|c|}
\hline & \multicolumn{6}{|c|}{ Men $(n=23,703)$} & & \multicolumn{6}{|c|}{ Women $(n=36,279)$} & \\
\hline & \multicolumn{2}{|c|}{$18-50$ Years $(n=14,890)$} & \multicolumn{2}{|c|}{$51-70$ Years $(n=8144)$} & \multicolumn{2}{|c|}{$>70$ Years $(n=669)$} & & \multicolumn{2}{|c|}{$18-50$ Years $(n=24,361)$} & \multicolumn{2}{|c|}{$51-70$ Years $(n=11,185)$} & \multicolumn{2}{|c|}{$>70$ Years $(n=733)$} & \\
\hline & Median & $\begin{array}{l}\text { 25th-75th } \\
\text { Percentile }\end{array}$ & Median & $\begin{array}{l}\text { 25th-75th } \\
\text { Percentile }\end{array}$ & Median & $\begin{array}{l}\text { 25th-75th } \\
\text { Percentile }\end{array}$ & & Median & $\begin{array}{l}\text { 25th-75th } \\
\text { Percentile }\end{array}$ & Median & $\begin{array}{l}\text { 25th-75th } \\
\text { Percentile }\end{array}$ & Median & $\begin{array}{l}\text { 25th-75th } \\
\text { Percentile }\end{array}$ & \\
\hline Soy products (g) & 0 & $0-0$ & 0 & $0-0$ & 0 & $0-0$ & $* * * a$ & 0 & $0-0$ & 0 & $0-0$ & 0 & $0-0$ & $* * * a c$ \\
\hline Sweets (g) & 37 & $19-60$ & 30 & $4-50$ & 31 & $16-48$ & $* * * a b$ & 27 & $14-45$ & 20 & $10-35$ & 21 & $10-34$ & $* * * a b$ \\
\hline Tea (g) & 89 & $11-232$ & 116 & $18-241$ & 232 & $116-465$ & $* * * a b c$ & 232 & $89-465$ & 232 & $116-465$ & 348 & $161-465$ & $* * * a b c$ \\
\hline Vegetables (g) & 128 & $87-181$ & 136 & 93-190 & 135 & 93-180 & $* * * a$ & 143 & 100-199 & 162 & $118-217$ & 152 & 109-200 & $* * * a b c$ \\
\hline Water $(\mathrm{g})$ & 279 & $107-557$ & 279 & $107-418$ & 279 & $139-418$ & $* * * a b$ & 386 & $139-579$ & 418 & $193-557$ & 418 & $161-557$ & $* * * a$ \\
\hline
\end{tabular}

${ }^{*}$ Significant difference between categories of age. ${ }^{*} p<0.05,{ }^{* * *} p<0.001$. a: significant difference between $18-50$ and $51-70$ years, b: significant difference between $18-50$ and $>70$ years, $c$ : significant difference between $51-70$ and $>70$ years.

Table 8. Daily food intake, categorised into food groups, per known SES category, obtained from the Flower FFQ $(n=58.971)$.

\begin{tabular}{|c|c|c|c|c|c|c|c|c|c|c|c|c|c|c|}
\hline & \multicolumn{6}{|c|}{ Men $(n=23,296)$} & & \multicolumn{6}{|c|}{ Women $(n=35,675)$} & \\
\hline & \multicolumn{2}{|c|}{ Low SES $(n=6590)$} & \multicolumn{2}{|c|}{$\begin{array}{c}\text { Moderate } \\
\text { SES }(n=8563)\end{array}$} & \multicolumn{2}{|c|}{ High SES $(n=8143)$} & & \multicolumn{2}{|c|}{ Low SES $(n=10,500)$} & \multicolumn{2}{|c|}{$\begin{array}{c}\text { Moderate SES } \\
(n=14,314)\end{array}$} & \multicolumn{2}{|c|}{ High SES $(n=10,861)$} & \\
\hline & Median & $\begin{array}{l}\text { 25th-75th } \\
\text { Percentile }\end{array}$ & Median & $\begin{array}{l}\text { 25th-75th } \\
\text { Percentile }\end{array}$ & Median & $\begin{array}{l}\text { 25th-75th } \\
\text { Percentile }\end{array}$ & & Median & $\begin{array}{l}\text { 25th-75th } \\
\text { Percentile }\end{array}$ & Median & $\begin{array}{l}\text { 25th-75th } \\
\text { Percentile }\end{array}$ & Median & $\begin{array}{l}\text { 25th-75th } \\
\text { Percentile }\end{array}$ & \\
\hline Alcoholic beverages (g) & 107 & $33-229$ & 107 & $36-227$ & 107 & $39-208$ & - & 21 & $0-76$ & 27 & $4-71$ & 27 & $4-71$ & $* * * a b c$ \\
\hline Artificially sweetened beverages (g) & 7 & $0-80$ & 11 & $0-92$ & 11 & $0-72$ & - & 11 & $0-72$ & 13 & $0-95$ & 13 & $0-95$ & $* * * a c$ \\
\hline Bread (g) & 165 & $123-213$ & 166 & $124-211$ & 153 & 113-201 & $* * * b c$ & 118 & $85-148$ & 117 & $83-149$ & 117 & $83-149$ & - \\
\hline Cake and cookies (g) & 31 & $18-48$ & 31 & $18-48$ & 30 & $17-47$ & $* * \mathrm{bc}$ & 31 & $18-47$ & 31 & $18-47$ & 31 & $18-47$ & $* * * b c$ \\
\hline Cheese (g) & 26 & $14-44$ & 25 & $13-42$ & 26 & $14-43$ & $* * a c$ & 23 & $13-40$ & 22 & $12-39$ & 22 & $12-39$ & $* * * a c$ \\
\hline Coffee $(\mathrm{g})$ & 465 & $348-697$ & 465 & $348-397$ & 465 & $348-697$ & $* b$ & 465 & $232-581$ & 348 & $116-465$ & 348 & $116-465$ & $* * * a b c$ \\
\hline Dairy (g) & 288 & $177-423$ & 294 & $185-431$ & 291 & $178-426$ & $* * * a c$ & 288 & $178-418$ & 284 & $172-417$ & 284 & $172-417$ & $* * * \mathrm{bc}$ \\
\hline Eggs (g) & 14 & $7-18$ & 9 & $7-18$ & 9 & $7-18$ & $* * * a b c$ & 7 & $4-18$ & 7 & $4-18$ & 7 & $4-18$ & $* * * a b$ \\
\hline
\end{tabular}


Table 8. Cont.

\begin{tabular}{|c|c|c|c|c|c|c|c|c|c|c|c|c|c|c|}
\hline & \multicolumn{6}{|c|}{ Men $(n=23,296)$} & & \multicolumn{6}{|c|}{ Women $(n=35,675)$} & \\
\hline & \multicolumn{2}{|c|}{ Low SES $(n=6590)$} & \multicolumn{2}{|c|}{$\begin{array}{c}\text { Moderate } \\
\text { SES }(n=8563)\end{array}$} & \multicolumn{2}{|c|}{ High SES $(n=8143)$} & & \multicolumn{2}{|c|}{ Low SES $(n=10,500)$} & \multicolumn{2}{|c|}{$\begin{array}{l}\text { Moderate SES } \\
\quad(n=14,314)\end{array}$} & \multicolumn{2}{|c|}{ High SES $(n=10,861)$} & \\
\hline & Median & $\begin{array}{l}\text { 25th-75th } \\
\text { Percentile }\end{array}$ & Median & $\begin{array}{l}\text { 25th-75th } \\
\text { Percentile }\end{array}$ & Median & $\begin{array}{l}\text { 25th-75th } \\
\text { Percentile }\end{array}$ & & Median & $\begin{array}{l}\text { 25th-75th } \\
\text { Percentile }\end{array}$ & Median & $\begin{array}{l}\text { 25th-75th } \\
\text { Percentile }\end{array}$ & Median & $\begin{array}{l}\text { 25th-75th } \\
\text { Percentile }\end{array}$ & \\
\hline Fat, oils and sauces (g) & 58 & $38-84$ & 59 & $39-84$ & 53 & $35-76$ & $* * * \mathrm{bc}$ & 39 & $25-58$ & 41 & $27-59$ & 41 & $27-59$ & $* * * \mathrm{ac}$ \\
\hline Fish (g) & 11 & $3-20$ & 12 & $4-21$ & 16 & $6-25$ & $* * * \mathrm{bc}$ & 11 & 2-19 & 11 & 2-19 & 11 & 2-19 & $* * * \mathrm{bc}$ \\
\hline Fruits (g) & 101 & $41-202$ & 100 & $42-201$ & 111 & $52-205$ & $* * * \mathrm{bc}$ & 137 & $65-207$ & 110 & $52-202$ & 110 & $52-202$ & $* * * a b c$ \\
\hline Legumes (g) & 7 & $0-18$ & 7 & $0-16$ & 7 & $0-16$ & $* * * a b$ & 4 & $0-16$ & 4 & $0-11$ & 4 & $0-11$ & $* * * a b c$ \\
\hline Meat $(\mathrm{g})$ & 86 & $64-110$ & 89 & $66-111$ & 83 & $62-105$ & $* * * a b c$ & 70 & $46-92$ & 72 & $50-95$ & 72 & $50-95$ & $* * * a b c$ \\
\hline Nuts and seeds (g) & 9 & $3-19$ & 10 & $4-21$ & 12 & $5-22$ & $* * * a b c$ & 6 & $2-14$ & 7 & $3-14$ & 7 & $3-14$ & $* * * a b c$ \\
\hline Pasta (g) & 19 & $11-32$ & 19 & $13-32$ & 20 & $13-45$ & $* * * a b c$ & 13 & $8-20$ & 19 & $12-26$ & 19 & $12-26$ & $* * * a b c$ \\
\hline Potatoes (g) & 100 & $55-149$ & 97 & $58-147$ & 86 & $48-122$ & ${ }^{* * *} \mathrm{bc}$ & 73 & $45-106$ & 72 & $45-105$ & 72 & $45-105$ & $* * * \mathrm{bc}$ \\
\hline $\begin{array}{l}\text { Probiotics and drinks lowering } \\
\text { cholesterol and blood pressure (g) }\end{array}$ & 0 & $0-0$ & 0 & $0-0$ & 0 & $0-0$ & $* * * a b c$ & 0 & $0-0$ & 0 & $0-0$ & 0 & $0-0$ & $* * * a b c$ \\
\hline Rice (g) & 15 & $2-26$ & 20 & $8-31$ & 21 & $11-39$ & $* * * a b c$ & 15 & $4-21$ & 16 & $6-25$ & 16 & $6-25$ & $* * * a b c$ \\
\hline Savoury snacks (g) & 26 & $12-46$ & 34 & $19-54$ & 33 & $17-52$ & $* * * a b c$ & 18 & $8-33$ & 25 & $13-42$ & 25 & $13-42$ & $* * * a b c$ \\
\hline Soft drinks (g) & 35 & $0-126$ & 42 & $3-136$ & 26 & $0-94$ & $* * * a b c$ & 0 & $0-47$ & 13 & $0-90$ & 13 & $0-90$ & $* * * a b c$ \\
\hline Soup (g) & 36 & $22-72$ & 36 & $22-72$ & 36 & $22-89$ & - & 36 & $22-72$ & 36 & $22-67$ & 36 & $22-67$ & - \\
\hline Soy products $(\mathrm{g})$ & 0 & $0-0$ & 0 & $0-0$ & 0 & $0-0$ & $* * * a b c$ & 0 & $0-0$ & 0 & $0-0$ & 0 & $0-0$ & $* * * a b c$ \\
\hline Sweets (g) & 34 & $17-58$ & 36 & $18-59$ & 33 & $17-54$ & $* * * a b c$ & 23 & $11-40$ & 26 & $13-43$ & 26 & $13-43$ & $* * * a b c$ \\
\hline Tea $(\mathrm{g})$ & 89 & $0-232$ & 89 & $11-232$ & 134 & $36-348$ & $* * * a b c$ & 232 & $80-348$ & 232 & $89-465$ & 232 & $89-465$ & $* * * a b c$ \\
\hline Vegan products other than soy $(\mathrm{g})$ & 0 & $0-0$ & 0 & $0-0$ & 0 & $0-0$ & $* * * a b c$ & 0 & $0-0$ & 0 & $0-0$ & 0 & $0-0$ & $* * * a b c$ \\
\hline Vegetables (g) & 122 & $81-171$ & 126 & $86-178$ & 146 & $102-200$ & $* * * a b c$ & 137 & $96-188$ & 143 & 101-197 & 143 & 101-197 & $* * * a b c$ \\
\hline Water $(\mathrm{g})$ & 279 & $107-557$ & 279 & $107-557$ & 279 & $107-418$ & - & 418 & 193-697 & 418 & $161-579$ & 418 & $161-579$ & $* * * a b c$ \\
\hline
\end{tabular}

${ }^{*}$ Significant difference between categories of SES. ${ }^{*} p<0.05,{ }^{* *} p<0.01,{ }^{* * *} p<0.001$. a: significant difference between low and moderate SES, b: significant difference between low and high SES, c: significant difference between moderate and high SES. 
Table 9. Daily food intake, categorised into food groups, per known BMI category, obtained from the Flower FFQ $(n=59.981)$

\begin{tabular}{|c|c|c|c|c|c|c|c|c|c|c|c|c|c|c|}
\hline & \multicolumn{6}{|c|}{ Men $(n=23,703)$} & & \multicolumn{6}{|c|}{ Women $(n=36,278)$} & \\
\hline & \multicolumn{2}{|c|}{$\begin{array}{l}\text { Normal Weight } \\
\quad(n=8875)\end{array}$} & \multicolumn{2}{|c|}{$\begin{array}{l}\text { Overweight } \\
(n=11,690)\end{array}$} & \multicolumn{2}{|c|}{ Obesity $(n=3138)$} & & \multicolumn{2}{|c|}{$\begin{array}{l}\text { Normal Weight } \\
\quad(n=18,608)\end{array}$} & \multicolumn{2}{|c|}{$\begin{array}{l}\text { Overweight } \\
(n=12,198)\end{array}$} & \multicolumn{2}{|c|}{ Obesity $(n=5472)$} & \\
\hline & Median & $\begin{array}{l}\text { 25th-75th } \\
\text { Percentile }\end{array}$ & Median & $\begin{array}{l}\text { 25th-75th } \\
\text { Percentile }\end{array}$ & Median & $\begin{array}{l}\text { 25th-75th } \\
\text { Percentile }\end{array}$ & & Median & $\begin{array}{l}\text { 25th-75th } \\
\text { Percentile }\end{array}$ & Median & $\begin{array}{l}\text { 25th-75th } \\
\text { Percentile }\end{array}$ & Median & $\begin{array}{l}\text { 25th-75th } \\
\text { Percentile }\end{array}$ & \\
\hline Alcoholic beverages (g) & 105 & $36-214$ & 109 & $38-223$ & 99 & $27-217$ & $* * * a b c$ & 35 & $7-90$ & 29 & $4-87$ & 13 & $0-56$ & ${ }^{* * *} \mathrm{abc}$ \\
\hline Artificially sweetened beverages (g) & 0 & $0-54$ & 13 & $0-92$ & 29 & $0-143$ & $* *$ abc & 7 & $0-54$ & 13 & $0-93$ & 29 & $0-139$ & ***abc \\
\hline Bread $(\mathrm{g})$ & 171 & $132-216$ & 154 & $114-204$ & 149 & 109-200 & $* * * a b c$ & 119 & $86-151$ & 113 & $81-145$ & 116 & $82-147$ & $* * * a b$ \\
\hline Cake and cookies (g) & 32 & $19-50$ & 30 & $17-47$ & 27 & $15-43$ & $* * * a b c$ & 31 & $18-47$ & 30 & $17-46$ & 29 & $16-44$ & $* * * a b$ \\
\hline Cheese (g) & 25 & $13-42$ & 26 & $14-43$ & 26 & $14-43$ & **ab & 23 & $13-40$ & 23 & $13-40$ & 22 & $13-39$ & ${ }^{*} \mathrm{C}$ \\
\hline Coffee (g) & 465 & $241-581$ & 465 & $348-697$ & 465 & $348-697$ & $* * * a b$ & 348 & $116-465$ & 348 & $232-581$ & 348 & $161-581$ & $* * * a b$ \\
\hline Dairy (g) & 295 & $186-431$ & 292 & $182-427$ & 276 & $162-407$ & $* * * \mathrm{bc}$ & 281 & $166-409$ & 285 & $175-418$ & 282 & $168-413$ & $* * a$ \\
\hline Eggs (g) & 9 & $7-18$ & 14 & $7-18$ & 14 & $7-18$ & $* * * a b c$ & 7 & $4-18$ & 7 & $4-18$ & 9 & $4-18$ & $* * * a b$ \\
\hline Fat, oils and sauces (g) & 59 & $40-84$ & 56 & $37-80$ & 53 & $34-77$ & $* * * a b c$ & 42 & $27-60$ & 39 & $25-57$ & 37 & $24-54$ & $* * * a b c$ \\
\hline Fish $(\mathrm{g})$ & 12 & $4-21$ & 13 & $4-22$ & 14 & $4-23$ & $* * * a b$ & 12 & $3-20$ & 12 & $4-21$ & 12 & $3-20$ & ${ }^{* *} \mathrm{C}$ \\
\hline Fruit juice (g) & 27 & $0-107$ & 21 & $0-96$ & 21 & $0-96$ & $* * * a b c$ & 21 & $0-96$ & 21 & $0-54$ & 13 & $0-54$ & $* * * a b c$ \\
\hline Legumes $(\mathrm{g})$ & 7 & $0-16$ & 7 & $0-16$ & 7 & $0-16$ & - & 4 & $0-11$ & 4 & $0-11$ & 4 & $0-11$ & ${ }^{* * *} \mathrm{bc}$ \\
\hline Meat (g) & 83 & 61-106 & 86 & $65-108$ & 92 & 68-115 & $* * * a b c$ & 68 & $41-92$ & 72 & 49-94 & 75 & $55-97$ & $* * * a b c$ \\
\hline Nuts and seeds (g) & 11 & $5-22$ & 11 & $5-21$ & 8 & $3-18$ & $* * * a b c$ & 7 & $3-15$ & 7 & $3-14$ & 6 & $2-12$ & ***abc \\
\hline Pasta (g) & 20 & 13-39 & 19 & $12-32$ & 19 & $11-28$ & $* *$ abc & 19 & $12-32$ & 16 & $8-26$ & 13 & $8-20$ & ${ }^{* * *} \mathrm{abc}$ \\
\hline Potatoes (g) & 97 & $55-143$ & 95 & $53-136$ & 90 & $47-132$ & $* *$ abc & 71 & $40-104$ & 72 & $40-104$ & 68 & 39-104 & - \\
\hline $\begin{array}{l}\text { Probiotics and drinks lowering } \\
\text { cholesterol and blood pressure (g) }\end{array}$ & 0 & $0-0$ & 0 & $0-0$ & 0 & $0-0$ & $* * * a b c$ & 0 & $0-0$ & 0 & $0-0$ & 0 & $0-0$ & $* * * a b$ \\
\hline Ready-made products (g) & 32 & $12-52$ & 31 & $2-50$ & 30 & $1-50$ & ${ }^{* * *} \mathrm{ab}$ & 23 & $6-36$ & 16 & $1-35$ & 18 & $1-36$ & ***ab \\
\hline Rice (g) & 20 & $9-34$ & 17 & $6-31$ & 16 & $4-27$ & $* * * a b c$ & 16 & $8-26$ & 15 & $5-24$ & 15 & $4-24$ & $* * * a b c$ \\
\hline Savoury snacks (g) & 33 & $17-53$ & 30 & $15-50$ & 29 & $15-50$ & $* * * a b$ & 23 & $12-40$ & 21 & $10-38$ & 21 & $10-38$ & $* * * a b$ \\
\hline
\end{tabular}


Table 9. Cont.

\begin{tabular}{|c|c|c|c|c|c|c|c|c|c|c|c|c|c|c|}
\hline & \multicolumn{6}{|c|}{ Men $(n=23,703)$} & & \multicolumn{6}{|c|}{ Women $(n=36,278)$} & \\
\hline & \multicolumn{2}{|c|}{$\begin{array}{l}\text { Normal Weight } \\
\quad(n=8875)\end{array}$} & \multicolumn{2}{|c|}{$\begin{array}{l}\text { Overweight } \\
(n=11,690)\end{array}$} & \multicolumn{2}{|c|}{ Obesity $(n=3138)$} & & \multicolumn{2}{|c|}{$\begin{array}{l}\text { Normal Weight } \\
\quad(n=18,608)\end{array}$} & \multicolumn{2}{|c|}{$\begin{array}{l}\text { Overweight } \\
(n=12,198)\end{array}$} & \multicolumn{2}{|c|}{ Obesity $(n=5472)$} & \\
\hline & Median & $\begin{array}{l}\text { 25th-75th } \\
\text { Percentile }\end{array}$ & Median & $\begin{array}{l}\text { 25th-75th } \\
\text { Percentile }\end{array}$ & Median & $\begin{array}{l}\text { 25th-75th } \\
\text { Percentile }\end{array}$ & & Median & $\begin{array}{l}\text { 25th-75th } \\
\text { Percentile }\end{array}$ & Median & $\begin{array}{l}\text { 25th-75th } \\
\text { Percentile }\end{array}$ & Median & $\begin{array}{l}\text { 25th-75th } \\
\text { Percentile }\end{array}$ & \\
\hline Soft drinks (g) & 42 & $5-134$ & 28 & $0-104$ & 26 & $0-126)$ & $* * * a b$ & 13 & $0-67$ & 5 & $0-52$ & 0 & $0-57$ & $* * * a b$ \\
\hline Soup (g) & 36 & $22-89$ & 36 & $22-89$ & 36 & $22-72$ & - & 36 & $22-45$ & 36 & $22-72$ & 36 & $22-72$ & $* * * a b$ \\
\hline Soy products (g) & 0 & $0-0$ & 0 & $0-0$ & 0 & $0-0$ & $* * * a b c$ & 0 & $0-0$ & 0 & $0-0$ & 0 & $0-0$ & $* * * a b c$ \\
\hline Tea $(\mathrm{g})$ & 116 & $18-322$ & 116 & $11-232$ & 80 & $0-232$ & $* * * a b c$ & 241 & $116-465$ & 232 & $89-465$ & 232 & $80-465$ & $* * * a b c$ \\
\hline Vegan products other than soy (g) & 0 & $0-0$ & 0 & $0-0$ & 0 & $0-0$ & $* * * a b c$ & 0 & $0-0$ & 0 & $0-0$ & 0 & $0-0$ & $* * * a b c$ \\
\hline Vegetables (g) & 134 & $93-189$ & 129 & $87-181$ & 129 & $86-180$ & $* * * a b$ & 148 & 106-205 & 149 & $106-205$ & 147 & $102-203$ & ${ }^{*} \mathrm{~b}$ \\
\hline Water $(\mathrm{g})$ & 279 & $107-418$ & 279 & $107-482$ & 289 & $139-557$ & $* * * a b c$ & 289 & $139-557$ & 418 & $193-697$ & 418 & $193-697$ & $* * * a b c$ \\
\hline
\end{tabular}

${ }^{*}$ Significant difference between categories of BMI. ${ }^{*} p<0.05,{ }^{* *} p<0.01,{ }^{* * *} p<0.001$. a: significant difference between normal weight and overweight, b: significant difference between normal weight and obesity, c: significant difference between overweight and obesity. 


\section{Discussion}

In this article, we describe dietary intake among participants of the Lifelines cohort study at baseline. Median energy intake, as well as the intake of macronutrients, was higher in men than in women and decreased with increasing categories of age and BMI. No striking differences in energy and macronutrient intake were observed for different categories of SES. In regard to the intake levels stratified for age, SES and BMI, an exception to the above concerns alcohol intake. Alcohol intake was highest in the middle age category, not in the lowest age category, in both men and women. Among different SES and BMI categories, differences were observed in women: alcohol intake was lowest in the low SES category and highest in the lowest BMI category. Regarding micronutrients, the intake was higher in men than in women, except for vitamin C. Among different age categories, different intake of micronutrients was observed, but no clear trend was observed: for some micronutrients the intake was higher among higher age categories compared to lower age categories, whereas for other micronutrients the opposite was observed. No striking differences in micronutrient intake were observed among different categories of SES and BMI. Intake of most food groups differed between men and women, as well as between different categories of age, SES and BMI.

\subsection{Generalisability}

The study population comprised mainly inhabitants of the northern three provinces of the Netherlands. The population in the north of the Netherlands has a homogeneous composition and low migration rates relative to other parts of the Netherlands and is therefore highly suitable for a long-lasting follow-up study such as the Lifelines cohort study [9]. To obtain an impression of the generalisability of the data, we compared the results of the Lifelines population in the current article with results from the Dutch National Food Consumption Survey (DNFCS) [20], which is compiled from a representative sample ( $n=2106 ; 1055$ men, 1051 women) of the general Dutch population. Data on dietary intake in the DNFCS are presented by age categories, which are different from the age categories in the current article. Here, we describe data for the age category 18-50 years in the present population and for the category 31-50 years in the DNFCS, but comparisons are applicable to other age categories as well. Compared to the DNFCS, the present population had a slightly lower median intake of energy (2476 vs. $2647 \mathrm{kcal}$ for men; $1892 \mathrm{vs.} 1956 \mathrm{kcal}$ for women). The intake of En\% from carbohydrates and fat were slightly higher (for carbohydrates 46 vs. $43 \mathrm{En} \%$ for men and 46 vs. $45 \mathrm{En} \%$ for women; for fats 37 vs. $35 \mathrm{En} \%$ for men and 37 vs. $34 \mathrm{En} \%$ for women), whereas the intake of En\% from protein was similar (15 En\% for men and $16 \mathrm{En} \%$ for women), and the intake of En\% from alcohol was lower (2.0 vs. $3.8 \mathrm{En} \%$ for men and 0.9 vs. $1.2 \mathrm{En} \%$ for women).

A comparison regarding intake of food groups between the present population and the DNFCS is more difficult to make, because within the DNFCS, food items were categorised into 17 food groups, and for the current article, food items were categorised into 30 food groups. Moreover, data on dietary intake in the DNFCS were collected using duplicate $24 \mathrm{~h}$ dietary recalls, which provide detailed information on dietary intake at two specific days, whereas in the Lifelines cohort study, dietary intake in the past month was assessed using an FFQ, which provides primarily information on food consumption patterns over time.

Because of this difference in dietary assessment method, we also compared our results to nutrient intake data obtained with a general FFQ, namely, data in the National Dietary Assessment Reference Database (NDARD) for the Dutch population ( $n=1647$; 857 men, 790 women), which was set up to serve as a reference database for new dietary assessment methods [21]. Data on energy and macronutrient intake in the NDARD are in the same range as in the present population and the DNFCS. It should be noted that NDARD participants lived in a relatively small part of the Netherlands around the city of Wageningen and had a higher SES compared to the general Dutch population, the DNFCS and Lifelines participants. 
It is important to note that a majority of Lifeline participants who completed the heart FFQ at the first assessment did not complete all three petal FFQs at subsequent assessments.

Comparison of participants who completed the total Flower FFQ and who did not complete it showed that completers were a little older, had a higher SES, smoked less and were less physically active compared to non-completers. Participants who completed the total Flower FFQ may live healthier or may be more conscious about their health and are therefore more likely to complete all the questionnaires, which means that some selection bias occurred. However, in the present study population, a large variation in age, SES, smoking behaviour and physical activity still exists. Together with the high degree of comparability with data from the DNFCS and the NDARD, we conclude that data on dietary intake of the Lifelines population in the current article are generalisable to the general Dutch population.

\subsection{Opportunities of Lifelines Data and Importance of Stratification}

The large sample size of the Lifelines cohort and its heterogeneity in participant characteristics provide the opportunity to perform well-powered stratified analyses in studies on associations between dietary intake and the development of chronic diseases and healthy aging. To study these associations, stratification is important to control for confounding factors and effect modifiers, such as sex, age, SES and BMI [22,23]. In this article, we presented dietary intake for men and women separately, as well as for different categories of age, SES and BMI. Regarding energy and macronutrient intake, we observed a decrease in intake with both increasing age categories and increasing BMI categories, whereas no striking differences were observed between different categories of SES. The observation within different categories of BMI seems paradoxical; however, it is well known that a higher BMI is associated with misreporting, which could be explained by the tendency of participants to providing socially desirable answers [24]. Another explanation may be that participants with a higher BMI followed a calorie-restricted diet more often than participants with a lower BMI. In men, the percentages of participants that followed such a diet were 0.6, 2.0 and 5.0 for the normal weight, overweight and obese categories, respectively. In women, these respective percentages were 2.7, 7.5 and 11.5. Regarding intake of food groups, differences were observed between different categories of age, SES and BMI. This underlines the importance of stratification in research on dietary intake.

\subsection{Strengths and Limitations}

All self-reporting methods are prone to several types of error such as recall bias or the tendency to provide socially desirable answers [25]. An FFQ is not the best method to evaluate absolute nutrient intake and adequacy of nutrient intake. A specific limitation of an FFQ is that single foods are grouped into groups of food items, wherein the variation of reported intake may be underestimated. This results in a smaller distribution of nutrient intake, and consequently an underestimation of the prevalence rate of (in)adequate intake. Therefore, calculating and interpreting such prevalence rates should be done with caution. However, an FFQ is a reliable method to rank participants to their intake levels [26,27], which is also true for the specific Flower FFQ [10]. In epidemiologic studies on associations of dietary intake with diseases or health status, such as the Lifelines cohort study, ranking of participants according to their intake levels is usually more relevant than absolute levels of intake. Moreover, FFQs are the cheapest and most feasible method to assess food consumption patterns over a long time, which is another reason for their usefulness is epidemiologic studies. For the participants, however, an FFQ may be time-consuming and therefore considered burdensome to complete. This may result in the return of incomplete questionnaires and less valid answers at the end compared to the beginning of the questionnaire. As the Flower FFQ consists of four questionnaires that are administered at different time points, experienced burden and risk of bias may be lower for this FFQ than for a general FFQ. To illustrate, comparison of the time used to complete a regular FFQ and the Flower FFQ showed that completion of a regular FFQ took on average 43 min, and 
completion of the heart FFQ and the first, second and third petal took on average 24, 9, 8 and $9 \mathrm{~min}$, respectively, adding up to a total of $50 \mathrm{~min}$ [10]. For the Lifelines cohort study, the different questionnaires were administered at different time points within a period of five years. Although stable food consumption patterns over time are assumed [11], changes in food consumption patterns may have occurred within these five years.

\section{Conclusions}

In conclusion, data on dietary intake obtained from the Flower FFQ among participants of the unique Lifelines cohort study are quite extensive and generalisable to the general Dutch population. As such, highly valuable dietary intake data are available in the Lifelines database to study associations between dietary intake and the development of chronic diseases and healthy aging.

Supplementary Materials: The following are available online at https://www.mdpi.com/article/10 .3390/nu14010048/s1, Table S1: Classification of 212 food items into 30 food groups.

Author Contributions: E.M.B.-B., C.W.M.P. and J.H.M.d.V. developed the Flower FFQ for the Lifelines cohort study; A.M.B. analysed the data and all authors interpreted the results; A.M.B. wrote the manuscript and E.M.B.-B., C.W.M.P., J.H.M.d.V. and E.J.M.F. critically reviewed it. All authors have read and agreed to the published version of the manuscript.

Funding: The Lifelines initiative has been made possible by subsidy from the Dutch Ministry of Health, Welfare and Sport, the Dutch Ministry of Economic Affairs, the University Medical Center Groningen (UMCG), Groningen University and the Provinces in the North of The Netherlands (Drenthe, Friesland, Groningen).

Institutional Review Board Statement: The Lifelines cohort study is conducted according to the principles of the Declaration of Helsinki and in accordance with the research code of the University Medical Center Groningen (UMCG). The Lifelines cohort study is approved by the medical ethical committee of the UMCG, The Netherlands.

Informed Consent Statement: Informed consent was obtained from all subjects involved in the study.Data availability Statement: Data may be obtained from a third party and are not publicly available. Researchers can apply to use the Lifelines data used in this study. More information about how to request Lifelines data and the conditions of use can be found on their website (https: // www.lifelines.nl/researcher/how-to-apply) (accessed on 1 November 2021).

Acknowledgments: The authors wish to acknowledge the services of the Lifelines cohort study, the contributing research centres delivering data to Lifelines and all study participants.

Conflicts of Interest: The authors declare no conflict of interest.

\section{References}

1. GBD 2017 Diet Collaborators. Health Effects of Dietary Risks in 195 Countries, 1990-2017: A Systematic Analysis for the Global Burden of Disease Study 2017. Lancet 2019, 393, 1958-1972. [CrossRef]

2. World Health Organization. Global Health Risks: Mortality and Burden of Disease Attributable to Selected Major Risks; World Health Organization: Geneva, Switzerland, 2009.

3. Key, T.J.; Bradbury, K.E.; Perez-Cornago, A.; Sinha, R.; Tsilidis, K.K.; Tsugane, S. Diet, nutrition, and cancer risk: What do we know and what is the way forward? BMJ 2020, 368, m511. [CrossRef] [PubMed]

4. Neuenschwander, M.; Ballon, A.; Weber, K.S.; Norat, T.; Aune, D.; Schwingshackl, L.; Schlesinger, S. Role of diet in type 2 diabetes incidence: Umbrella review of meta-analyses of prospective observational studies. BMJ 2019, 366, 12368. [CrossRef]

5. Pan, A.; Lin, X.; Hemler, E.; Hu, F.B. Diet and Cardiovascular Disease: Advances and Challenges in Population-Based Studies. Cell Metab. 2018, 27, 489-496. [CrossRef]

6. Franzago, M.; Santurbano, D.; Vitacolonna, E.; Stuppia, L. Genes and Diet in the Prevention of Chronic Diseases in Future Generations. Int. J. Mol. Sci. 2020, 21, 2633. [CrossRef]

7. O'Grady, J.; Shanahan, F. Macronutrients, microbiome and precision nutrition. Curr. Opin. Gastroenterol. 2020, 37, $145-151$. [CrossRef] [PubMed]

8. Satija, A.; Yu, E.; Willett, W.C.; Hu, F.B. Understanding nutritional epidemiology and its role in policy. Adv. Nutr. 2015, 6, 5-18. [CrossRef] [PubMed] 
9. Scholtens, S.; Smidt, N.; Swertz, M.A.; Bakker, S.J.L.; Dotinga, A.; Vonk, J.M.; Van Dijk, F.; Van Zon, S.K.R.; Wijmenga, C.; Wolffenbuttel, B.H.; et al. Cohort Profile: LifeLines, a three-generation cohort study and biobank. Int. J. Epidemiol. 2015, 44, 1172-1180. [CrossRef] [PubMed]

10. Brouwer-Brolsma, E.M.; Perenboom, C.; Sluik, D.; van de Wiel, A.; Geelen, A.; Feskens, E.J.; de Vries, J.H. Development and external validation of the 'Flower-FFQ': A food frequency questionnaire designed for the Lifelines Cohort Study. Public Health Nutr. 2021; 1-12, (Online ahead of print.).

11. Goldbohm, R.A.; van't Veer, P.; van den Brandt, P.A.; van't Hof, M.A.; Brants, H.A.; Sturmans, F.; Hermus, R.J. Reproducibility of a food frequency questionnaire and stability of dietary habits determined from five annually repeated measurements. Eur. J. Clin. Nutr. 1995, 49, 420-429. [PubMed]

12. NEVO-Tabel, Dutch Food Composition Table 2011/Version 3; RIVM: Bilthoven, The Netherlands, 2011.

13. Willett, W.C. Nutritional Epidemiology, 3rd ed.; Oxford University Press: New York, NY, USA, 2013.

14. Rhee, J.J.; Sampson, L.; Cho, E.; Hughes, M.D.; Hu, F.B.; Willett, W.C. Comparison of Methods to Account for Implausible Reporting of Energy Intake in Epidemiologic Studies. Am. J. Epidemiol. 2015, 181, 225-233. [CrossRef] [PubMed]

15. Available online: www.gezondheidsraad.nl (accessed on 1 September 2021).

16. Vart, P.; Gansevoort, R.T.; Coresh, J.; Reijneveld, S.; Bültmann, U. Socioeconomic Measures and CKD in the United States and The Netherlands. Clin. J. Am. Soc. Nephrol. 2013, 8, 1685-1693. [CrossRef] [PubMed]

17. Wendel-Vos, G.C.; Schuit, A.J.; Saris, W.H.; Kromhout, D. Reproducibility and relative validity of the short questionnaire to assess health-enhancing physical activity. J. Clin. Epidemiol. 2003, 56, 1163-1169. [CrossRef]

18. Ainsworth, B.E.; Haskell, W.L.; Herrmann, S.D.; Meckes, N.; Bassett, D.R., Jr.; Tudor-Locke, C.; Greer, J.L.; Vezina, J.; Whitt-Glover, M.C.; Leon, A.S.; et al. Compendium of Physical Activities: A second update of codes and MET values. Med. Sci. Sport. Exerc. 2011, 43, 1575-1581. [CrossRef] [PubMed]

19. World Health Organization. Obesity: Preventing and Managing the Global Epidemic: Report of a WHO Consultation; WHO Technical Report Series; World Health Organization: Geneva, Switzerland, 1999.

20. Rossum, C.T.M.; Fransen, H.P.; Verkaik-Kloosterman, J.; Buurma-Rethans, E.J.M.; Ocké, M.C. Dutch National Food Consumption Survey 2007-2010: Diet of Children Aged 7 to 69 Years; RIVM: Bilthoven, The Netherlands, 2011.

21. Brouwer-Brolsma, E.M.; Streppel, M.T.; Van Lee, L.; Geelen, A.; Sluik, D.; van de Wiel, A.M.; De Vries, J.H.; Van't Veer, P.; Feskens, E.J. A National Dietary Assessment Reference Database (NDARD) for the Dutch Population: Rationale behind the Design. Nutrients 2017, 9, 1136. [CrossRef] [PubMed]

22. Jager, K.; Zoccali, C.; MacLeod, A.; Dekker, F. Confounding: What it is and how to deal with it. Kidney Int. 2008, 73, 256-260. [CrossRef] [PubMed]

23. Corraini, P.; Olsen, M.; Pedersen, L.; Dekkers, O.M.; Vandenbroucke, J.P. Effect modification, interaction and mediation: An overview of theoretical insights for clinical investigators. Clin. Epidemiol. 2017, 9, 331-338. [CrossRef] [PubMed]

24. Trijsburg, L.; Geelen, A.; Hollman, P.C.; Hulshof, P.J.; Feskens, E.J.; Veer, P.V.; Boshuizen, H.C.; de Vries, J.H. BMI was found to be a consistent determinant related to misreporting of energy, protein and potassium intake using self-report and duplicate portion methods. Public Health Nutr. 2017, 20, 598-607. [CrossRef] [PubMed]

25. Naska, A.; Lagiou, A.; Lagiou, P. Dietary assessment methods in epidemiological research: Current state of the art and future prospects. F1000Research 2017, 6, 926. [CrossRef] [PubMed]

26. Siebelink, E.; Geelen, A.; de Vries, J.H.M. Self-reported energy intake by FFQ compared with actual energy intake to maintain body weight in 516 adults. Br. J. Nutr. 2011, 106, 274-281. [CrossRef] [PubMed]

27. Streppel, M.T.; de Vries, J.H.; Meijboom, S.; Beekman, M.; De Craen, A.J.; Slagboom, P.E.; Feskens, E.J. Relative validity of the food frequency questionnaire used to assess dietary intake in the Leiden Longevity Study. Nutr. J. 2013, 12, 75-78. [CrossRef] [PubMed] 